\title{
Regiospecific Synthesis of 5- and 6-Acylated Naphtho[1,2-b]ben- zofurans via Intramolecular Alkyne Carbonyl Metathesis
}

\author{
Maloy Nayak \\ Dileep Kumar Singh \\ Ikyon Kim* \\ College of Pharmacy and Yonsei Institute of Pharmaceuti- \\ cal Sciences, Yonsei University, 85 Songdogwahak-ro, \\ Yeonsu-gu, Incheon, 21983, Republic of Korea \\ ikyonkim@yonsei.ac.kr
}

Received: 25.10 .2016

Accepted after revision: 21.11.2016

Published online: 15.12 .2016

DOI: 10.1055/s-0036-1588120; Art ID: ss-2016-f0747-op

Abstract Straightforward access to various 5- and 6-acylated naphtho[1,2-b]benzofurans was achieved by successive Sonogashira coupling and intramolecular alkyne carbonyl metathesis to assemble the central aromatic $C$ ring of naphtho[1,2-b]benzofurans regiospecifically substituted with an acyl group at the $\mathrm{C} 5$ or $\mathrm{C} 6$ position.

Key words naphtho[1,2-b]benzofuran, Sonogashira coupling, alkyne carbonyl metathesis, intramolecular reaction, regiospecific reaction

Intramolecular alkyne carbonyl metathesis (IACM) of an alkyne and a carbonyl spatially arranged within one molecular skeleton provides an efficient way to make carbo- or heterocycles with an enone functional group (Scheme 1, a). ${ }^{1}$ Recently, we have employed this strategy for the synthesis of benzo-fused indolizines, pyrrolo[1,2-a]quinolines, ${ }^{2}$ as well as natural products, such as brazilin, deguelin, and munduserone, ${ }^{3}$ constructing the pyridine ring in the former and 3- or 4-acylchromene in the latter.

As an extension, we decided to investigate IACM-based modular synthesis of polyaromatic heterocycles, ${ }^{4}$ an important class of chemicals in the area of pharmaceuticals and functional materials. Since IACM typically allows the regiospecific introduction of an acyl moiety in the newly formed ring systems, we hoped to construct polyheterocycles with an acyl group positioned at a specific site, which is quite difficult to achieve by any other synthetic methods such as intermolecular Friedel-Crafts acylation. As part of our continued interest in polyfunctionalized benzofurans ${ }^{5}$ we wish to describe here a synthetic approach to naphtho[1,2-b]benzofurans ${ }^{6}$ with an acyl unit at the C5 or C6 position $^{7}$ through IACM (Scheme 1, b). As 2-arylbenzofuran,

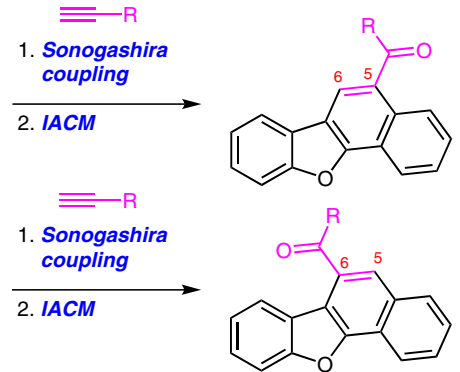

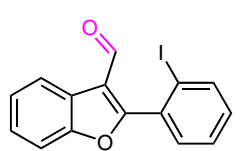

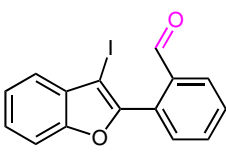

1-acylnaphthalene, and 2-acylnaphthalene have been employed as key pharmacophores in the area of small-molecule drug discovery, we expected that these two hybrid structures would be useful in the discovery of new interesting biological activities. ${ }^{8}$ Retrosynthetically, 2-arylbenzofurans, 2 and 5, having both alkyne and aldehyde groups were required for this purpose. In turn, we expected that Sonogashira cross-coupling of $\mathbf{3}$ and $\mathbf{6}$ with terminal alkynes would give rise to $\mathbf{2}$ and $\mathbf{5}$, respectively.
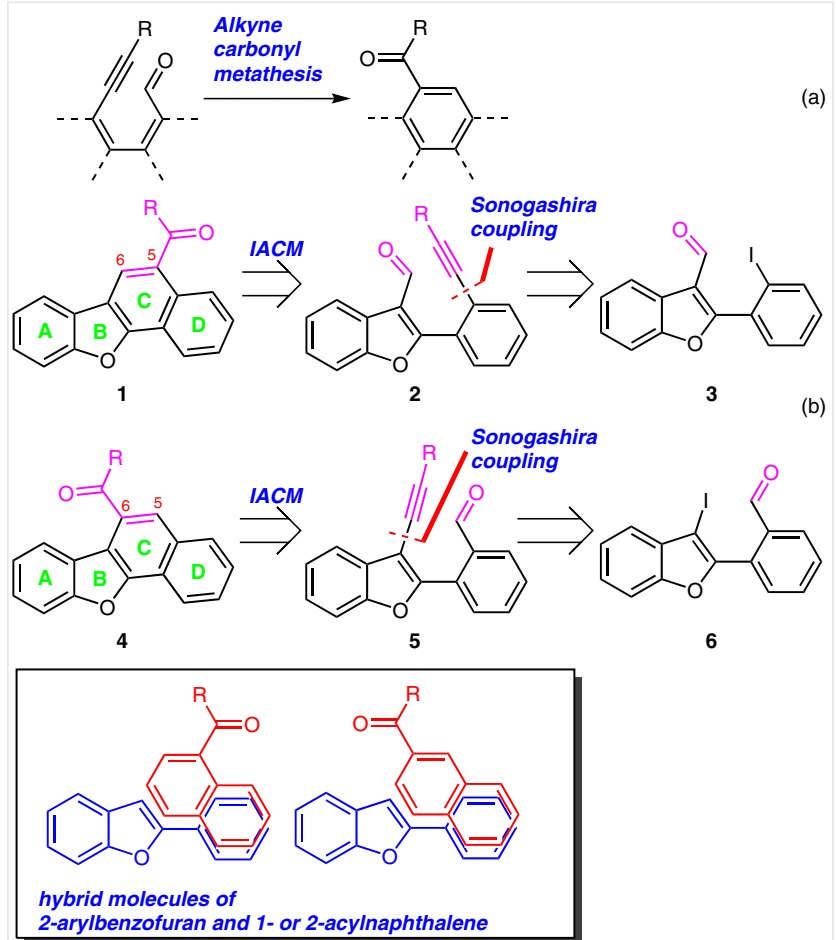

Scheme 1 Synthetic plans 

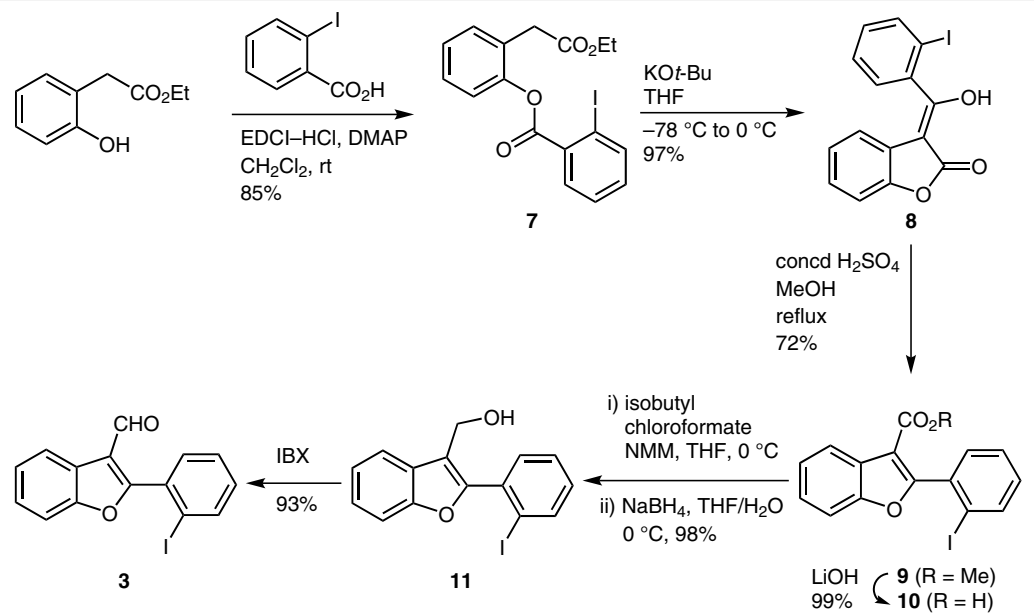

Scheme 2 Synthesis of 3

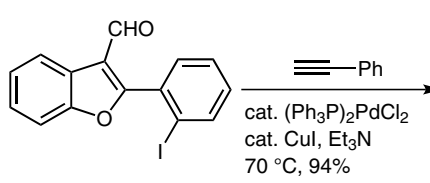

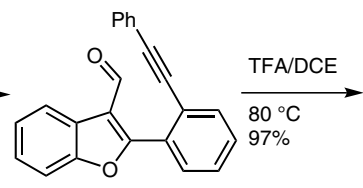

2a

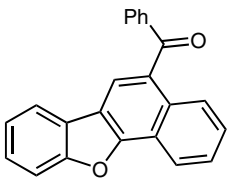

1a

Scheme 3 Sonogashira coupling/IACM of 3

To validate our proposal, preparation of the requisite $\mathbf{3}$ was first attempted. After several unsuccessful attempts, we finally resorted to Chi's protocol (Scheme 2). ${ }^{9}$ First, ethyl (2-hydroxyphenyl)acetate was coupled with 2-iodobenzoic acid under modified Steglich esterification conditions ${ }^{10}$ to give ester 7. Exposure of $\mathbf{7}$ to excess $t$-BuOK resulted in $\beta$ keto ester $\mathbf{8}$ in its enol form in excellent yield, which was smoothly converted into methyl 2-(2-iodophenyl)benzofuran-3-carboxylate (9) by treatment of $\mathrm{MeOH}$ and concentrated $\mathrm{H}_{2} \mathrm{SO}_{4}$. The ester moiety in $\mathbf{9}$ was transformed into the desired aldehyde to furnish $\mathbf{3}$ in good overall yield by a three-step sequence: hydrolysis, $\mathrm{NaBH}_{4}$ reduction of the mixed anhydride formed in situ, and IBX oxidation.

When 3 was subjected to Sonogashira coupling conditions with phenylacetylene, 2a was isolated in 94\% yield (Scheme 3). After screening of several ACM catalyst conditions, we were pleased to find that heating of $\mathbf{2} \mathbf{a}$ in TFA/DCE at $80{ }^{\circ} \mathrm{C}$ for 1 hour deliver the desired product 1a in excellent yield.

With these optimized conditions in hand, substrate scope was examined with different terminal alkynes (Table 1). Sonogashira cross-coupling of $\mathbf{3}$ with several (hetero)arylalkynes proceeded well to give the corresponding alkynes $\mathbf{2}$ in excellent yields. Subsequent IACM of $\mathbf{2}$ under the optimized conditions furnished the desired 5-acylnaphtho[1,2-b]benzofurans in good yields except for $\mathbf{2 f}$ (en- try 5). The formation of $\mathbf{1 f}^{\prime}$ can be interpreted as a consequence of domino alkyne carbonyl metathesis/Nazarov cyclization. ${ }^{11}$

Next, 6 was synthesized for the synthesis of 6 -acylnaphtho[1,2-b]benzofurans 4 (Scheme 4). Sonogashira coupling of 1-ethynyl-2-methoxybenzene with 2-iodobenzaldehyde afforded the corresponding product 12 in 78\% yield. To our disappointment, however, no desired 3-iodobenzofuran 6 was observed from the reaction of $\mathbf{1 2}$ under several iodocyclization conditions, which might be ascribed to the competitive participation of the formyl group in $\mathbf{1 2}$ in iodine-

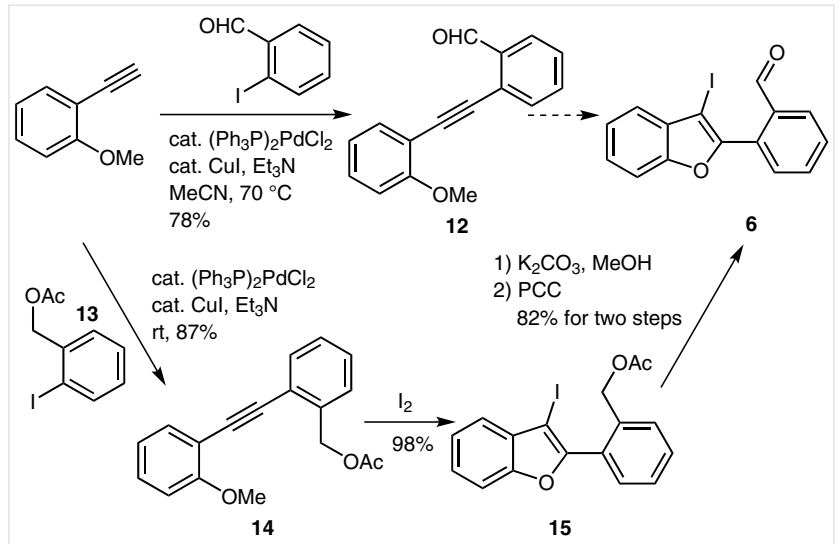

Scheme 4 Synthesis of 6 
Table 1 Synthesis of Diverse 5-Acylnaphtho[1,2-b]benzofurans ${ }^{a}$

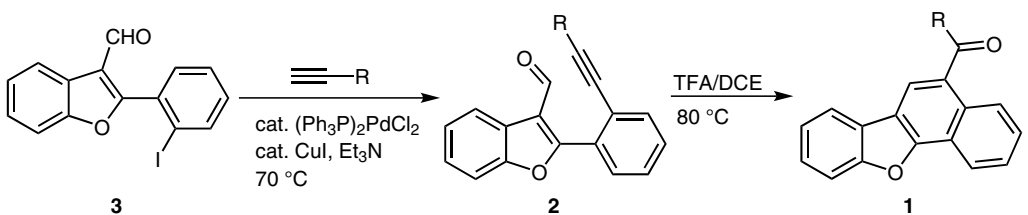

\begin{tabular}{|c|c|c|c|c|}
\hline Entry & Alkyne & 2 (yield,, \%) & Product & 1 (yield, b \%) \\
\hline 1 & $\equiv$ & $\mathbf{2 b}(98)$ & & $1 \mathbf{b}(97)$ \\
\hline 2 & $\overline{\bar{\equiv}}$ & 2c (96) & & 1c $(90)$ \\
\hline 3 & & 2d (94) & & 1d (93) \\
\hline 4 & & $2 e(98)$ & & 1e (93) \\
\hline 5 & $\bar{\equiv}$ & $2 f(75)$ & & $1 \mathbf{f}^{\prime}(68)$ \\
\hline 6 & $\equiv$ & $\mathbf{2 g}(95)$ & & $\mathbf{1 g}(87)$ \\
\hline
\end{tabular}

a Reaction conditions: 1.3 (0.14 mmol), terminal alkyne (0.16 mmol), $\left(\mathrm{Ph}_{3} \mathrm{P}\right)_{2} \mathrm{PdCl}_{2}\left(0.1\right.$ equiv), Cul (0.05 equiv), Et $\mathrm{N}_{3}(2 \mathrm{~mL}), 70^{\circ} \mathrm{C}, 12 \mathrm{~h} ; 2.2(0.1 \mathrm{mmol})$ TFA/DCE (1:2, $3 \mathrm{~mL}) ; 80^{\circ} \mathrm{C} 1 \mathrm{~h}$.

b Isolated yield. 
2066

Synthesis

M. Nayak et al.

Paper

mediated cyclization. ${ }^{12}$ These difficulties in preparing 6 were overcome by replacing the coupling partner by 2 -iodobenzyl acetate (13). Thus, when 1-ethynyl-2-methoxybenzene was treated with 13 in the presence of $\left(\mathrm{Ph}_{3} \mathrm{P}\right)_{2} \mathrm{PdCl}_{2}$ ( 0.1 equiv) and $\mathrm{CuI}$ (0.05 equiv) in $\mathrm{Et}_{3} \mathrm{~N}$ at room temperatore, the cross-coupled product $\mathbf{1 4}$ was obtained in excellent yield. Smooth iodocyclization took place upon exposure of $\mathbf{1 4}$ to $\mathrm{I}_{2}$ (4 equiv), affording the cyclized product $\mathbf{1 5}$ in $98 \%$ yield. Deacetylation of $\mathbf{1 5}$ and subsequent PCC oxideton gave 6, setting the stage for sequential Sonogashira coupling/IACM for the synthesis of 6-acylnaphtho[1,2b] benzofuran.

As shown in Table 2, Sonogashira coupling of $\mathbf{6}$ with phenylacetylene under the similar reaction conditions employed for the synthesis of $\mathbf{2}$ led to $\mathbf{5 a}$ in $\mathbf{9 2} \%$ yield (entry $\mathbf{1}$ ).
Again, subjection of $\mathbf{5 a}$ in TFA/DCE at $80{ }^{\circ} \mathrm{C}$ for 1 hour produce the desired product $\mathbf{4 a}$ in excellent yield. The rearton scope was also investigated with different (hetero)aryland alkylalkynes under the same reaction sequence. Variours 6-acylnaphtho[1,2-b]benzofurans $\mathbf{4}$ were synthesized in good overall yields. The IACM reaction with a substrate bearing an electron-deficient aryl group on the alkyne $\mathbf{5 f}$ also worked well under these conditions to give $\mathbf{4 f}$ in $90 \%$ yield (entry 6). When $\mathbf{5 g}$ having a cyclohex-1-enyl group was exposed to TFA at $80^{\circ} \mathrm{C}$, hexacyclic compound $\mathbf{4 g}^{\prime}$ was obtained as the major product due to Nazarov reaction after IACM (entry 7).

Table 2 Synthesis of Diverse 6-Acylnaphtho[1,2-b]benzofurans ${ }^{a}$

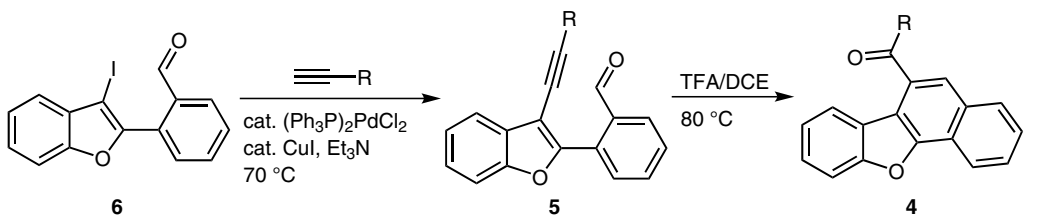

\begin{tabular}{ll}
\hline Entry & Alkyne \\
\hline 1 &
\end{tabular}

Sa (92)

2

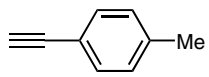

$\mathbf{5 b}(85)$

3

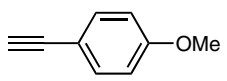

$5 c(83)$

4

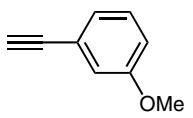

Product

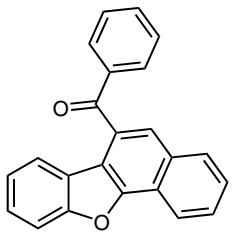

ab (95)
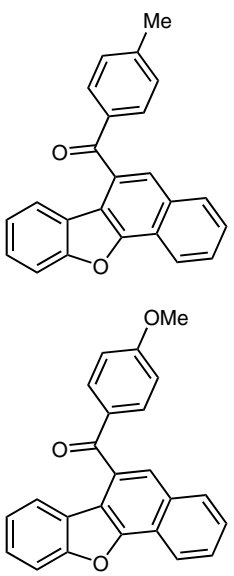

4a (90)

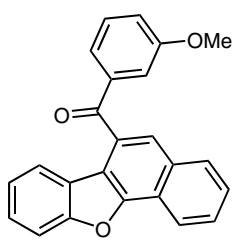

Ac (90)

4d (88)

(c) Georg Theme Verlag Stuttgart · New York - Synthesis 2017, 49, 2063-2073 
Table 2 (continued)

\begin{tabular}{|c|c|c|c|c|}
\hline Entry & Alkyne & 5 (yield, b \%) & Product & 4 (yield, $\left.{ }^{b} \%\right)$ \\
\hline 5 & & $5 e(76)$ & & $4 \mathbf{e}(89)$ \\
\hline 6 & & $5 \mathbf{f}(82)$ & & $\mathbf{4 f}(90)$ \\
\hline 7 & $\equiv$ & $\mathbf{5 g}(88)$ & & $\mathbf{4} \mathbf{g}^{\prime}(82)$ \\
\hline 8 & $\equiv$ & $5 h(96)$ & & 4h (97) \\
\hline 9 & $\equiv$ & $5 \mathbf{i}(96)$ & & $4 \mathbf{i}(87)$ \\
\hline
\end{tabular}

a Reaction conditions: 1.6 (0.14 mmol), terminal alkyne (0.16 mmol), $\left(\mathrm{Ph}_{3} \mathrm{P}\right)_{2} \mathrm{PdCl}_{2}\left(0.1\right.$ equiv), Cul (0.05 equiv), Et ${ }_{3} \mathrm{~N}(2 \mathrm{~mL}), 70{ }^{\circ} \mathrm{C}, 12 \mathrm{~h} ; 2.5(0.1 \mathrm{mmol})$, TFA/DCE (1:2, $3 \mathrm{~mL}) ; 80^{\circ} \mathrm{C}, 1 \mathrm{~h}$.

b Isolated yield.

In summary, we have established an expeditious route to 5- and 6-acylated naphtho[1,2-b]benzofurans, hybrid structures of 2-arylbenzofuran and 1- or 2-acylnaphthalene, by utilizing a sequential Sonogashira coupling/intramolecular alkyne carbonyl metathesis reaction where the benzene ring $C$ of this scaffold was formed with an acyl substituent at the specific position. Both aryl- and alkylalkynes were employed in this sequence to generate a wide variety of new 6-acylnaphtho[1,2-b]benzofurans, exhibiting good functional group tolerance. Application of this protocol for the synthesis of other polyaromatic heterocycles as well as evaluation of these compounds in biomedical sciences are underway in our laboratory.
Unless specified, all reagents and starting materials were purchased from commercial sources and used as received without purification. 'Concentrated' refers to the removal of volatile solvents via distillation using a rotary evaporator. 'Dried' refers to pouring onto, or passing through, anhyd $\mathrm{MgSO}_{4}$ followed by filtration. Flash chromatography was performed using silica gel (230-400 mesh) with hexanes, EtOAc, and $\mathrm{CH}_{2} \mathrm{Cl}_{2}$ as eluent. All reactions were monitored by TLC on 0.25 $\mathrm{mm}$ silica plates (F-254) visualizing with UV light. Melting points were measured using a capillary melting point apparatus. ${ }^{1} \mathrm{H}$ and ${ }^{13} \mathrm{C}$ NMR spectra were recorded on $400 \mathrm{MHz}$ NMR spectrometer. HRMS were measured with electrospray ionization (ESI) and Q-TOF mass analyzer. 


\section{2-(2-Ethoxy-2-oxoethyl)phenyl 2-Iodobenzoate (7)}

To a stirred solution of ethyl (2-hydroxyphenyl)acetate (1.2 g, 6.66 $\mathrm{mmol})$ in anhyd $\mathrm{CH}_{2} \mathrm{Cl}_{2}(25 \mathrm{~mL})$ were added 2-iodobenzoic acid (1.98 g, $7.99 \mathrm{mmol})$, EDCI-HCl (1.53 g, $7.99 \mathrm{mmol})$, and DMAP (163 mg, $1.33 \mathrm{mmol}$ ) at $\mathrm{rt}$ and the mixture was stirred overnight. The mixture was washed with $1 \mathrm{M} \mathrm{HCl}$ and water followed by sat. aq $\mathrm{NaHCO}_{3}$ solution. The organic layer was dried $\left(\mathrm{MgSO}_{4}\right)$ and concentrated in vacuo to yield the crude product which was purified by flash chromatography (silica gel, hexanes/EtOAc, 9:1) give 7 (2.33 g, 85\%) as a colorless liquid.

${ }^{1} \mathrm{H} \mathrm{NMR}\left(400 \mathrm{MHz}, \mathrm{CDCl}_{3}\right.$ ): $\delta=8.09(\mathrm{t}, J=8.1 \mathrm{~Hz}, 2 \mathrm{H}), 7.49(\mathrm{t}, J=7.6$ $\mathrm{Hz}, 1 \mathrm{H}), 7.35-7.39(\mathrm{~m}, 2 \mathrm{H}), 7.21-7.30(\mathrm{~m}, 3 \mathrm{H}), 4.08$ (q, $J=7.1 \mathrm{~Hz}, 2$ $\mathrm{H}), 3.64(\mathrm{~s}, 2 \mathrm{H}), 1.14(\mathrm{t}, J=7.1 \mathrm{~Hz}, 3 \mathrm{H})$.

${ }^{13} \mathrm{C}$ NMR $\left(100 \mathrm{MHz}, \mathrm{CDCl}_{3}\right): \delta=170.7,164.4,149.2,141.9,133.7$, 133.4, 131.7, 131.5, 128.7, 128.19, 126.8, 126.5, 122.6, 94.9, 61.1, 36.7, 14.2 .

HRMS (ESI-QTOF): $m / z[M+H]^{+}$calcd for $\mathrm{C}_{17} \mathrm{H}_{16} \mathrm{IO}_{4}$ : 411.0088; found: 411.0090 .

\section{3-[Hydroxy(2-iodophenyl)methylene]benzofuran-2(3H)-one (8)}

To a mixture of KOt-Bu (862 mg, $7.68 \mathrm{mmol})$ in anhyd THF $(40 \mathrm{~mL})$ under a $\mathrm{N}_{2}$ atmosphere was dropwise added a solution of 7 ( $2.1 \mathrm{~g}$, $5.12 \mathrm{mmol})$ in THF $(5 \mathrm{~mL})$ at $-78^{\circ} \mathrm{C}$. The mixture was stirred for 10 min at $-78{ }^{\circ} \mathrm{C}$, and then it was warmed to $0{ }^{\circ} \mathrm{C}$ and stirred for $2 \mathrm{~h}$ at this temperature. The mixture was quenched with $1 \mathrm{M} \mathrm{HCl}(20 \mathrm{~mL})$, diluted with EtOAc $(30 \mathrm{~mL})$, and washed with water $(2 \times 20 \mathrm{~mL})$. The organic layer was dried $\left(\mathrm{MgSO}_{4}\right)$ and concentrated in vacuo to yield the product ( $1.81 \mathrm{~g}, 97 \%$ ) as a greenish yellow solid, which was used for the next step without further purification; $\mathrm{mp} 129.4-131.5^{\circ} \mathrm{C}$.

${ }^{1} \mathrm{H} \mathrm{NMR}\left(400 \mathrm{MHz}, \mathrm{CDCl}_{3}\right): \delta=8.04(\mathrm{~d}, J=8.0 \mathrm{~Hz}, 1 \mathrm{H}), 7.55(\mathrm{t}, J=7.6$ $\mathrm{Hz}, 1 \mathrm{H}), 7.45(\mathrm{~d}, J=7.6 \mathrm{~Hz}, 1 \mathrm{H}), 7.30(\mathrm{~d}, J=7.6 \mathrm{~Hz}, 1 \mathrm{H}), 7.16-7.23(\mathrm{~m}$, $2 \mathrm{H}), 6.93(\mathrm{t}, J=7.2 \mathrm{~Hz}, 1 \mathrm{H}), 6.31(\mathrm{~d}, J=7.2 \mathrm{~Hz}, 1 \mathrm{H})$.

${ }^{13} \mathrm{C}$ NMR $\left(100 \mathrm{MHz}, \mathrm{CDCl}_{3}\right): \delta=173.4,171.2,151.1,140.3,138.1$, 132.3, 129.3, 128.9, 127.6, 124.2, 121.9, 120.2, 111.0, 100.1, 94.5.

HRMS (ESI-QTOF): $m / z$ [M + H] $]^{+}$calcd for $\mathrm{C}_{15} \mathrm{H}_{10} \mathrm{IO}_{3}$ : 364.9669; found: 364.9668 .

\section{Methyl 2-(2-Iodophenyl)benzofuran-3-carboxylate (9)}

To a stirred solution of $\mathbf{8}(1.3 \mathrm{~g}, 3.57 \mathrm{mmol})$ in anhyd $\mathrm{MeOH}(40 \mathrm{~mL})$ was slowly added concd $\mathrm{H}_{2} \mathrm{SO}_{4}(4.5 \mathrm{~mL})$ at r.t. The resulting mixture was refluxed for $24 \mathrm{~h}$, cooled to r.t., and concentrated under reduced pressure. The residue was diluted with EtOAc $(20 \mathrm{~mL})$ and washed with water $(2 \times 10 \mathrm{~mL})$ and sat. aq $\mathrm{NaHCO}_{3}$ solution $(2 \times 10 \mathrm{~mL})$. The combined organic layers were dried $\left(\mathrm{MgSO}_{4}\right)$ and concentrated in vacuo to yield the crude product which was purified by flash chromatography (silica gel, hexanes/EtOAc, 19:1) afforded $\mathbf{9}(970 \mathrm{mg}, 72 \%$ ) as a white solid; mp $99.5-101.6{ }^{\circ} \mathrm{C}$.

${ }^{1} \mathrm{H}$ NMR (400 MHz, $\left.\mathrm{CDCl}_{3}\right): \delta=8.13-8.15(\mathrm{~m}, 1 \mathrm{H}), 7.99(\mathrm{~d}, J=7.8 \mathrm{~Hz}, 1$ H), 7.56-7.58 (m, 1 H), 7.47-7.49 (m, 2 H), 7.40-7.43 (m, 2 H), 7.18$7.22(\mathrm{~m}, 1 \mathrm{H}), 3.83(\mathrm{~s}, 3 \mathrm{H})$.

${ }^{13} \mathrm{C}$ NMR $\left(100 \mathrm{MHz}, \mathrm{CDCl}_{3}\right): \delta=163.7,162.2,154.2,139.3,136.1$, 131.7, 131.4, 127.8, 125.9, 125.7, 124.4, 122.6, 111.6, 111.1, 98.3, 51.8. HRMS (ESI-QTOF): $m / z[M+\mathrm{H}]^{+}$calcd for $\mathrm{C}_{16} \mathrm{H}_{12} \mathrm{IO}_{3}$ : 378.9826; found: 378.9823 .

\section{2-(2-Iodophenyl)benzofuran-3-carboxylic Acid (10)}

To a stirred solution of $\mathbf{9}(1 \mathrm{~g}, 2.64 \mathrm{mmol})$ in $\mathrm{THF} / \mathrm{H}_{2} \mathrm{O}(1: 1,20 \mathrm{~mL})$ was added $\mathrm{LiOH} \cdot \mathrm{H}_{2} \mathrm{O}(555 \mathrm{mg}, 13.22 \mathrm{mmol})$. The mixture was stirred at $80{ }^{\circ} \mathrm{C}$ for $12 \mathrm{~h}$, and then it was cooled to r.t., acidified with $2 \mathrm{M} \mathrm{HCl}$, and extracted with EtOAc $(20 \mathrm{~mL})$. The organic layer was dried $\left(\mathrm{MgSO}_{4}\right)$ and concentrated in vacuo to yield the product $(960 \mathrm{mg}$, $99 \%$ ) as a white solid which was used for the next step without further purification; $\mathrm{mp} 194.7-196.2^{\circ} \mathrm{C}$.

${ }^{1} \mathrm{H}$ NMR $\left(400 \mathrm{MHz}\right.$, DMSO- $\left.d_{6}\right): \delta=8.07(\mathrm{~d}, J=6.7 \mathrm{~Hz}, 1 \mathrm{H}), 8.01(\mathrm{~d}$, $J=7.8 \mathrm{~Hz}, 1 \mathrm{H}), 7.70$ (d, $J=6.8 \mathrm{~Hz}, 1 \mathrm{H}), 7.52-7.59(\mathrm{~m}, 2 \mathrm{H}), 7.41-7.47$ (m, $2 \mathrm{H}), 7.30(\mathrm{t}, J=7.3 \mathrm{~Hz}, 1 \mathrm{H})$.

${ }^{13} \mathrm{C}$ NMR $\left(100 \mathrm{MHz}\right.$, DMSO- $\left.d_{6}\right): \delta=163.9,161.7,153.6,138.6,135.8$, 131.8, 131.7, 127.9, 125.9, 125.7, 124.4, 122.2, 111.6, 111.3, 99.2.

HRMS (ESI-QTOF): $m / z[\mathrm{M}+\mathrm{H}]^{+}$calcd for $\mathrm{C}_{15} \mathrm{H}_{10} \mathrm{IO}_{3}$ : 364.9669; found: 364.9668 .

\section{[2-(2-Iodophenyl)benzofuran-3-yl]methanol (11)}

To a stirred solution of $\mathbf{1 0}(960 \mathrm{mg}, 2.65 \mathrm{mmol})$ in THF $(20 \mathrm{~mL})$ at $0{ }^{\circ} \mathrm{C}$ were added isobutyl chloroformate $(0.414 \mathrm{~mL}, 3.18 \mathrm{mmol})$ and $\mathrm{N}$ methylmorpholine $(0.35 \mathrm{~mL}, 3.18 \mathrm{mmol})$. The resulting mixture was stirred for $2 \mathrm{~min}$, and the precipitate obtained was filtered through a pad of Celite and washed with THF ( $5 \mathrm{~mL})$. The combined filtrates were added to another round bottom flask and cooled to $0{ }^{\circ} \mathrm{C}$. To this solution was added dropwise $\mathrm{NaBH}_{4}(200 \mathrm{mg}, 5.30 \mathrm{mmol})$ dissolved in water $(0.5 \mathrm{~mL})$. The mixture was stirred for $5 \mathrm{~min}$ at $0{ }^{\circ} \mathrm{C}$, and then it was quenched with water $(10 \mathrm{~mL})$ and extracted with EtOAc $(3 \times 20$ $\mathrm{mL})$. The combined organic layers were dried $\left(\mathrm{MgSO}_{4}\right)$ and concentrated in vacuo to yield the crude product which was purified by flash chromatography (silica gel, hexanes/EtOAc, 4:1) to afford 11 (905 mg, 98\%) as an off-white solid; $\mathrm{mp} 98.3-100.4{ }^{\circ} \mathrm{C}$.

${ }^{1} \mathrm{H}$ NMR $\left(400 \mathrm{MHz}, \mathrm{CDCl}_{3}\right): \delta=8.00(\mathrm{~d}, J=7.9 \mathrm{~Hz}, 1 \mathrm{H}), 7.81(\mathrm{~d}, J=7.2$ $\mathrm{Hz}, 1 \mathrm{H}), 7.54(\mathrm{~d}, J=8.0 \mathrm{~Hz}, 1 \mathrm{H}), 7.44-7.48(\mathrm{~m}, 2 \mathrm{H}), 7.31-7.39$ (m, 2 H), 7.17-7.20 (m, $1 \mathrm{H}), 4.75$ (s, $2 \mathrm{H}), 1.81(\mathrm{~s}, 1 \mathrm{H})$.

${ }^{13} \mathrm{C}$ NMR $\left(100 \mathrm{MHz}, \mathrm{CDCl}_{3}\right): \delta=154.5,154.3,139.8,135.6,132.2$, 131.2, 128.2, 125.1, 123.2, 120.6, 117.0, 111.6, 99.1, 56.4.

HRMS (ESI-QTOF): $\mathrm{m} / z$ [M $+\mathrm{Na}]^{+}$calcd for $\mathrm{C}_{15} \mathrm{H}_{11} \mathrm{NaIO}_{2}: 372.9696$; found: 372.9697 .

\section{2-(2-Iodophenyl)benzofuran-3-carbaldehyde (3)}

To a stirred solution of $\mathbf{1 1}(990 \mathrm{mg}, 2.83 \mathrm{mmol})$ in EtOAc $(20 \mathrm{~mL})$ was added IBX (2.4 g, $8.49 \mathrm{mmol})$. The mixture was refluxed for $3 \mathrm{~h}$, and then it was cooled to r.t., filtered through a pad of Celite, and washed with EtOAc. The filtrate was washed with sat. aq $\mathrm{NaHCO}_{3}(3 \times 10 \mathrm{~mL})$ and water $(2 \times 10 \mathrm{~mL})$. The combined organic layers were dried $\left(\mathrm{MgSO}_{4}\right)$ and concentrated in vacuo to give the crude product which was purified by flash chromatography (silica gel, hexanes/EtOAc, 9:1) to afford 3 (914 mg, 93\%) as a white solid; $\mathrm{mp} 104.9-106.9{ }^{\circ} \mathrm{C}$.

${ }^{1} \mathrm{H} \mathrm{NMR}\left(400 \mathrm{MHz}, \mathrm{CDCl}_{3}\right): \delta=9.98(\mathrm{~s}, 1 \mathrm{H}), 8.29(\mathrm{~d}, J=8.0 \mathrm{~Hz}, 1 \mathrm{H})$, 8.05-8.07 (m, $1 \mathrm{H}), 7.59-7.61$ ( $\mathrm{m}, 1 \mathrm{H}), 7.53-7.54$ ( $\mathrm{m}, 2 \mathrm{H}), 7.41-7.47$ (m, $2 \mathrm{H}), 7.26-7.30(\mathrm{~m}, 1 \mathrm{H})$.

${ }^{13} \mathrm{C}$ NMR $\left(100 \mathrm{MHz}, \mathrm{CDCl}_{3}\right): \delta=186.6,166.8,154.4,140.3,133.9$, 132.6, 132.3, 128.3, 126.4, 125.1, 124.4, 122.9, 118.9, 111.6, 98.2.

HRMS (ESI-QTOF): $m / z$ [M + H] $]^{+}$calcd for $\mathrm{C}_{15} \mathrm{H}_{10} \mathrm{IO}_{2}$ : 348.9720; found: 348.9723 .

\section{2-[2-(Phenylethynyl)phenyl]benzofuran-3-carbaldehyde (2a); Typical Procedure}

To a mixture of 3 (50 mg, $0.14 \mathrm{mmol})$ in $\mathrm{Et}_{3} \mathrm{~N}(2 \mathrm{~mL})$ were added phenylacetylene $(0.018 \mathrm{~mL}, 0.16 \mathrm{mmol}),\left(\mathrm{Ph}_{3} \mathrm{P}\right)_{2} \mathrm{PdCl}_{2}(10 \mathrm{mg}, 0.014$ $\mathrm{mmol})$, and $\mathrm{CuI}(1.33 \mathrm{mg}, 0.007 \mathrm{mmol})$. The mixture was stirred at $70{ }^{\circ} \mathrm{C}$ for $12 \mathrm{~h}$, and then it was concentrated in vacuo to yield the 
crude product. Purification by flash chromatography (silica gel, hexanes/EtOAc, 49:1) afforded 2a (43.5 mg, 94\%) as an off-white solid; mp $102.3-104.6^{\circ} \mathrm{C}$.

${ }^{1} \mathrm{H}$ NMR $\left(400 \mathrm{MHz}, \mathrm{CDCl}_{3}\right): \delta=10.26(\mathrm{~s}, 1 \mathrm{H}), 8.31-8.32(\mathrm{~m}, 1 \mathrm{H})$, 7.70-7.75 (m, $2 \mathrm{H}), 7.49-7.58$ ( $\mathrm{m}, 3 \mathrm{H}), 7.40-7.42$ ( $\mathrm{m}, 2 \mathrm{H}), 7.31-7.33$ ( $\mathrm{m}, 2 \mathrm{H}), 7.25-7.27$ ( $\mathrm{m}, 3 \mathrm{H})$.

${ }^{13} \mathrm{C}$ NMR $\left(100 \mathrm{MHz}, \mathrm{CDCl}_{3}\right): \delta=187.0,164.2,154.7,133.6,131.7$, $130.8,130.8,130.4,128.9,128.6,128.5,126.2,125.1,124.9,124.1$, $122.9,122.4,118.7,111.4,94.0,87.4$.

HRMS (ESI-QTOF): $m / z$ [M + H] calcd for $\mathrm{C}_{23} \mathrm{H}_{15} \mathrm{O}_{2}$ : 323.1067; found: 323.1066 .

\section{2-[2-(p-Tolylethynyl)phenyl]benzofuran-3-carbaldehyde (2b)}

White solid; yield: $47.5 \mathrm{mg}$ (98\%); $\mathrm{mp} 112.2-114.4{ }^{\circ} \mathrm{C}$.

${ }^{1} \mathrm{H}$ NMR $\left(400 \mathrm{MHz}, \mathrm{CDCl}_{3}\right): \delta=10.28(\mathrm{~s}, 1 \mathrm{H}), 8.33-8.34(\mathrm{~m}, 1 \mathrm{H})$, 7.72-7.76 (m, $2 \mathrm{H}), 7.49-7.60$ (m, $3 \mathrm{H}), 7.42-7.43(\mathrm{~m}, 2 \mathrm{H}), 7.23$ (d, $J=7.6 \mathrm{~Hz}, 2 \mathrm{H}), 7.08$ (d, $J=7.6 \mathrm{~Hz}, 2 \mathrm{H}), 2.32(\mathrm{~s}, 3 \mathrm{H})$.

${ }^{13} \mathrm{C}$ NMR $\left(100 \mathrm{MHz}, \mathrm{CDCl}_{3}\right): \delta=187.0,164.2,154.7,139.2,133.4$, 131.6, 130.8, 130.7, 130.3, 129.3, 128.4, 126.1, 125.2, 124.9, 124.3, 122.9, 119.3, 118.6, 111.3, 94.3, 86.9, 21.7.

HRMS (ESI-QTOF): $m / z$ [M + H] calcd for $\mathrm{C}_{24} \mathrm{H}_{17} \mathrm{O}_{2}$ : 337.1223; found: 337.1226 .

\section{2-\{2-[(4-Methoxyphenyl)ethynyl]phenyl\}benzofuran-3-carbalde-} hyde (2c)

Pale yellow solid; yield: $48.6 \mathrm{mg}$ (96\%); $\mathrm{mp} 96.2-98.6{ }^{\circ} \mathrm{C}$.

${ }^{1} \mathrm{H}$ NMR $\left(400 \mathrm{MHz}, \mathrm{CDCl}_{3}\right): \delta=10.27(\mathrm{~s}, 1 \mathrm{H}), 8.31-8.32(\mathrm{~m}, 1 \mathrm{H}), 7.70$ (br s, $2 \mathrm{H}), 7.48-7.58(\mathrm{~m}, 3 \mathrm{H}), 7.40-7.41(\mathrm{~m}, 2 \mathrm{H}), 7.25(\mathrm{~d}, J=8.0 \mathrm{~Hz}, 2$ $\mathrm{H}), 6.78(\mathrm{~d}, J=8.0 \mathrm{~Hz}, 2 \mathrm{H}), 3.77(\mathrm{~s}, 3 \mathrm{H})$.

${ }^{13} \mathrm{C}$ NMR $\left(100 \mathrm{MHz}, \mathrm{CDCl}_{3}\right): \delta=187.1,164.3,160.2,154.7,133.3$, 133.2, 130.7, 130.1, 128.2, 126.1, 125.1, 124.9, 124.5, 122.9, 118.6, 114.5, 114.2, 111.3, 94.2, 86.3, 55.4.

HRMS (ESI-QTOF): $m / z$ [M $+\mathrm{H}]^{+}$calcd for $\mathrm{C}_{24} \mathrm{H}_{17} \mathrm{O}_{3}$ : 353.1172; found: 353.1174.

\section{2-\{2-[(3-Methoxyphenyl)ethynyl]phenyl\}benzofuran-3-carbalde- hyde (2d)}

Off-white solid; yield: $47.5 \mathrm{mg}$ (94\%); $\mathrm{mp} 161.4-163.2^{\circ} \mathrm{C}$.

${ }^{1} \mathrm{H}$ NMR (400 MHz, $\left.\mathrm{CDCl}_{3}\right): \delta=10.28(\mathrm{~s}, 1 \mathrm{H}), 8.31-8.33(\mathrm{~m}, 1 \mathrm{H})$, 7.72-7.76 (m, $2 \mathrm{H}), 7.52-7.60(\mathrm{~m}, 3 \mathrm{H}), 7.41-7.43(\mathrm{~m}, 2 \mathrm{H}), 7.17$ (t, $J=7.6 \mathrm{~Hz}, 1 \mathrm{H}), 6.94(\mathrm{~d}, J=7.4 \mathrm{~Hz}, 1 \mathrm{H}), 6.83-6.86(\mathrm{~m}, 2 \mathrm{H}), 3.73$ (s, 3 $\mathrm{H})$.

${ }^{13} \mathrm{C}$ NMR $\left(100 \mathrm{MHz}, \mathrm{CDCl}_{3}\right): \delta=187.0,164.2,159.4,154.7,133.4$, 130.8, 130.8, 130.5, 129.6, 128.6, 126.2, 125.1, 124.9, 124.2, 124.1, $123.4,122.9,118.7,116.0,111.4,93.9,87.2,55.4$.

HRMS (ESI-QTOF): $m / z$ [M $+\mathrm{H}]^{+}$calcd for $\mathrm{C}_{24} \mathrm{H}_{17} \mathrm{O}_{3}: 353.1172$; found: 353.1178.

\section{2-[2-(Naphthalen-1-ylethynyl)phenyl]benzofuran-3-carbalde-} hyde (2e)

Yellow solid; yield: $52.5 \mathrm{mg}$ (98\%); $\mathrm{mp} 150.1-153.4^{\circ} \mathrm{C}$.

${ }^{1} \mathrm{H}$ NMR $\left(400 \mathrm{MHz}, \mathrm{CDCl}_{3}\right): \delta=10.28(\mathrm{~s}, 1 \mathrm{H}), 8.36-8.37(\mathrm{~m}, 1 \mathrm{H}), 7.94$ (d, $J=8.3 \mathrm{~Hz}, 1 \mathrm{H}), 7.89$ (d, $J=8.3 \mathrm{~Hz}, 1 \mathrm{H}), 7.79-7.80$ (m, $2 \mathrm{H}), 7.74$ (d, $J=7.4 \mathrm{~Hz}, 1 \mathrm{H}$ ), 7.53-7.63 (m, $4 \mathrm{H}), 7.37-7.47$ (m, $4 \mathrm{H}), 7.15-7.19$ (m, $1 \mathrm{H})$.
${ }^{13} \mathrm{C}$ NMR $\left(100 \mathrm{MHz}, \mathrm{CDCl}_{3}\right): \delta=186.9,164.7,154.8,133.7,133.1$, 133.1, 131.2, 130.9, 130.9, 130.2, 129.4, 128.6, 128.3, 126.9, 126.5, 126.3, 126.0, 125.3, 125.06, 125.0, 124.4, 122.9, 120.1, 119.1, 111.6, 92.4, 92.1.

HRMS (ESI-QTOF): $m / z$ [M $+\mathrm{H}]^{+}$calcd for $\mathrm{C}_{27} \mathrm{H}_{17} \mathrm{O}_{3}: 373.1223$; found: 373.1227.

\section{2-[2-(Cyclohex-1-en-1-ylethynyl)phenyl]benzofuran-3-carbalde- hyde (2f)}

Pale yellow solid; yield: $35.0 \mathrm{mg}$ (75\%); $\mathrm{mp} 103.1-105.2{ }^{\circ} \mathrm{C}$.

${ }^{1} \mathrm{H}$ NMR (400 MHz, $\left.\mathrm{CDCl}_{3}\right): \delta=10.20(\mathrm{~s}, 1 \mathrm{H}), 8.30-8.31(\mathrm{~m}, 1 \mathrm{H}), 7.67$ $(\mathrm{d}, J=7.2 \mathrm{~Hz}, 1 \mathrm{H}), 7.64-7.62(\mathrm{~d}, J=7.5 \mathrm{~Hz}, 1 \mathrm{H}), 7.55-7.57(\mathrm{~m}, 1 \mathrm{H})$, 7.40-7.51 (m, 4 H), 6.08 (s, 1 H), 2.02-2.06 (m, 4 H), 1.49-1.61 (m, 4 $\mathrm{H})$.

${ }^{13} \mathrm{C}$ NMR $\left(100 \mathrm{MHz}, \mathrm{CDCl}_{3}\right): \delta=187.1,164.3,154.6,136.8,133.3$, 130.6, 130.6, 130.1, 128.0, 126.0, 125.1, 124.8, 124.6, 122.9, 120.3, 118.5, 111.3, 96.0, 84.9, 28.7, 25.9, 22.2, 21.4.

HRMS (ESI-QTOF): $m / z$ [M $+\mathrm{H}]^{+}$calcd for $\mathrm{C}_{23} \mathrm{H}_{19} \mathrm{O}_{2}: 327.1380$; found: 327.1382 .

\section{2-[2-(Thiophen-3-ylethynyl)phenyl]benzofuran-3-carbaldehyde \\ (2g)}

Off-white solid; yield: $45.0 \mathrm{mg}$ (95\%); $\mathrm{mp} 93.5-95.8^{\circ} \mathrm{C}$.

${ }^{1} \mathrm{H}$ NMR $\left(400 \mathrm{MHz}, \mathrm{CDCl}_{3}\right): \delta=10.29(\mathrm{~s}, 1 \mathrm{H}), 8.33-8.34(\mathrm{~m}, 1 \mathrm{H})$, 7.72-7.74 (m, 2 H), 7.52-7.59 (m, $3 \mathrm{H}), 7.40-7.43$ (m, $3 \mathrm{H}), 7.23$ (s, 1 $\mathrm{H}), 7.01(\mathrm{~d}, J=4.6 \mathrm{~Hz}, 1 \mathrm{H})$.

${ }^{13} \mathrm{C}$ NMR $\left(100 \mathrm{MHz}, \mathrm{CDCl}_{3}\right): \delta=187.1,164.0,154.7,133.3,130.7$, 130.3, 129.7, 129.7, 128.5, 126.2, 125.6, 125.1, 124.9, 124.1, 122.9, 121.5, 118.6, 111.3, 89.3, 87.0.

HRMS (ESI-QTOF): $m / z$ [M + H] $]^{+}$calcd for $\mathrm{C}_{21} \mathrm{H}_{13} \mathrm{O}_{2} \mathrm{~S}: 329.0631$; found: 329.0632 .

\section{Naphtho[1,2-b]benzofuran-5-yl(phenyl)methanone (1a)}

To a solution of $\mathbf{2 a}(32 \mathrm{mg}, 0.1 \mathrm{mmol})$ in DCE $(2.0 \mathrm{~mL})$ was added TFA $(1.0 \mathrm{~mL})$ and the resulting solution was stirred at $80^{\circ} \mathrm{C}$ for $1 \mathrm{~h}$. Then the mixture was cooled down to r.t. and concentrated in vacuo to yield the crude product. Purification by flash chromatography (silica gel, hexanes/EtOAc, 49:1) afforded 1a (31 mg, 97\%) as an off-white solid; mp $167.4-168.9^{\circ} \mathrm{C}$.

${ }^{1} \mathrm{H} \mathrm{NMR}\left(400 \mathrm{MHz}, \mathrm{CDCl}_{3}\right): \delta=8.56(\mathrm{~d}, J=8.0 \mathrm{~Hz}, 1 \mathrm{H}), 8.33(\mathrm{~d}, J=8.2$ $\mathrm{Hz}, 1 \mathrm{H}), 8.17$ (s, $1 \mathrm{H}), 7.94-7.95$ (m, $3 \mathrm{H}), 7.69-7.77$ (m, $2 \mathrm{H}), 7.59-$ $7.66(\mathrm{~m}, 2 \mathrm{H}), 7.51-7.52(\mathrm{~m}, 3 \mathrm{H}), 7.39-7.43(\mathrm{~m}, 1 \mathrm{H})$.

${ }^{13} \mathrm{C}$ NMR $\left(100 \mathrm{MHz}, \mathrm{CDCl}_{3}\right): \delta=197.8,156.5,138.9,133.3,132.0$, 131.2, 130.7, 128.6, 127.5, 127.2, 127.0, 126.9, 124.7, 123.6, 122.1, $121.7,121.4,120.6,117.8,112.2$.

HRMS (ESI-QTOF): $m / z[\mathrm{M}+\mathrm{Na}]^{+}$calcd for $\mathrm{C}_{23} \mathrm{H}_{14} \mathrm{NaO}_{2}: 346.0886$; found: 346.0888 .

\section{Naphtho[1,2-b]benzofuran-5-yl(p-tolyl)methanone (1b)}

Off-white solid; yield: $33.0 \mathrm{mg}$ (97\%); $\mathrm{mp} 163.4-165.6^{\circ} \mathrm{C}$.

${ }^{1} \mathrm{H}$ NMR $\left(400 \mathrm{MHz}, \mathrm{CDCl}_{3}\right): \delta=8.55(\mathrm{~d}, J=8.0 \mathrm{~Hz}, 1 \mathrm{H}), 8.28(\mathrm{~d}, J=8.4$ $\mathrm{Hz}, 1 \mathrm{H}), 8.15(\mathrm{~s}, 1 \mathrm{H}), 7.95(\mathrm{~d}, J=7.2 \mathrm{~Hz}, 1 \mathrm{H}), 7.84(\mathrm{~d}, J=7.6 \mathrm{~Hz}, 2 \mathrm{H})$, 7.68-7.76 (m, $2 \mathrm{H}), 7.50-7.61(\mathrm{~m}, 2 \mathrm{H}), 7.40(\mathrm{t}, J=7.2 \mathrm{~Hz}, 1 \mathrm{H}), 7.30$ (d, $J=7.6 \mathrm{~Hz}, 2 \mathrm{H}), 2.46(\mathrm{~s}, 3 \mathrm{H})$.

${ }^{13} \mathrm{C}$ NMR $\left(100 \mathrm{MHz}, \mathrm{CDCl}_{3}\right): \delta=197.5,156.4,144.3,136.3,132.4$, $131.1,130.8,129.4,127.4,127.1,126.9,126.9,124.8,123.5,121.7$, $121.5,121.4,120.56,117.8,112.2,21.9$. 
HRMS (ESI-QTOF): $m / z[\mathrm{M}+\mathrm{Na}]^{+}$calcd for $\mathrm{C}_{24} \mathrm{H}_{16} \mathrm{NaO}_{2}: 359.0042$; found: 359.0043 .

\section{(4-Methoxyphenyl)(naphtho[1,2-b]benzofuran-5-yl)methanone \\ (1c)}

White solid; yield: $31.5 \mathrm{mg}$ (90\%); $\mathrm{mp} 154.2-156.4^{\circ} \mathrm{C}$.

${ }^{1} \mathrm{H}$ NMR (400 MHz, $\left.\mathrm{CDCl}_{3}\right): \delta=8.54(\mathrm{~d}, J=8.0 \mathrm{~Hz}, 1 \mathrm{H}), 8.22(\mathrm{~d}, J=8.3$ $\mathrm{Hz}, 1 \mathrm{H}), 8.14$ (s, $1 \mathrm{H}), 7.91-7.97$ (m, $3 \mathrm{H}), 7.67-7.76$ (m, $2 \mathrm{H}), 7.50-$ 7.60 (m, $2 \mathrm{H}), 7.39-7.43$ (m, $1 \mathrm{H}), 6.97$ (d, $J=8.5 \mathrm{~Hz}, 2 \mathrm{H}), 3.89(\mathrm{~s}, 3 \mathrm{H})$. ${ }^{13} \mathrm{C}$ NMR $\left(100 \mathrm{MHz}, \mathrm{CDCl}_{3}\right): \delta=196.5,163.9,156.4,153.5,133.0$, 132.7, 131.6, 131.1, 127.3, 127.1, 126.9, 126.9, 124.8, 123.5, 121.7, $121.4,120.9,120.5,117.9,113.9,112.1,55.7$.

HRMS (ESI-QTOF): $m / z[\mathrm{M}+\mathrm{Na}]^{+}$calcd for $\mathrm{C}_{24} \mathrm{H}_{16} \mathrm{NaO}_{3}: 375.0991$; found: 375.0994 .

\section{(3-Methoxyphenyl)(naphtho[1,2-b]benzofuran-5-yl)methanone (1d)}

Off-white solid; yield: $32.6 \mathrm{mg}$ (93\%); $\mathrm{mp} 147.3-149.6^{\circ} \mathrm{C}$.

${ }^{1} \mathrm{H} \mathrm{NMR}\left(400 \mathrm{MHz}, \mathrm{CDCl}_{3}\right): \delta=8.55(\mathrm{~d}, J=8.3 \mathrm{~Hz}, 1 \mathrm{H}), 8.32(\mathrm{~d}, J=8.5$ $\mathrm{Hz}, 1 \mathrm{H}), 8.17(\mathrm{~s}, 1 \mathrm{H}), 7.95(\mathrm{~d}, J=7.5 \mathrm{~Hz}, 1 \mathrm{H}), 7.69-7.77(\mathrm{~m}, 2 \mathrm{H})$, 7.50-7.63 (m, $3 \mathrm{H}), 7.35-7.43(\mathrm{~m}, 3 \mathrm{H}), 7.18(\mathrm{~d}, J=7.7 \mathrm{~Hz}, 1 \mathrm{H}), 3.87(\mathrm{~s}$, $3 \mathrm{H})$.

${ }^{13} \mathrm{C}$ NMR $\left(100 \mathrm{MHz}, \mathrm{CDCl}_{3}\right): \delta=197.6,159.9,156.5,153.8,140.3$, 132.1, 131.2, 129.6, 127.5, 127.2, 127.0, 126.9, 124.7, 123.8, 123.6, $122.0,121.7,121.4,120.6,120.0,117.8,114.3,112.2,55.7$.

HRMS (ESI-QTOF): $m / z$ [M $+\mathrm{H}]^{+}$calcd for $\mathrm{C}_{24} \mathrm{H}_{17} \mathrm{O}_{3}: 353.1172$; found: 353.1172 .

\section{Naphthalen-1-yl(naphtho[1,2-b]benzofuran-5-yl)methanone (1e)} Gray solid; yield: $34.5 \mathrm{mg}$ (93\%); $\mathrm{mp} 182.5-184.6{ }^{\circ} \mathrm{C}$.

${ }^{1} \mathrm{H}$ NMR (400 MHz, $\left.\mathrm{CDCl}_{3}\right): \delta=8.82(\mathrm{~d}, J=8.3 \mathrm{~Hz}, 1 \mathrm{H}), 8.57-8.59(\mathrm{~m}, 2$ $\mathrm{H}), 8.20(\mathrm{~s}, 1 \mathrm{H}), 8.07(\mathrm{~d}, J=8.1 \mathrm{~Hz}, 1 \mathrm{H}), 7.98-7.99(\mathrm{~m}, 1 \mathrm{H}), 7.81(\mathrm{~d}$, $J=7.1 \mathrm{~Hz}, 1 \mathrm{H}), 7.68-7.75$ (m, $4 \mathrm{H}), 7.59-7.62$ (m, $2 \mathrm{H}), 7.47-7.51$ (m, $2 \mathrm{H}), 7.35(\mathrm{t}, J=7.5 \mathrm{~Hz}, 1 \mathrm{H})$.

${ }^{13} \mathrm{C}$ NMR $\left(100 \mathrm{MHz}, \mathrm{CDCl}_{3}\right): \delta=199.3,156.4,154.5,137.8,134.1$, 133.0, 132.5, 131.5, 131.5, 130.2, 128.6, 128.1, 128.0, 127.3, 127.1, 127.0, 126.7, 126.0, 124.9, 124.6, 123.6, 121.8, 121.4, 120.6, 117.8, 112.1.

HRMS (ESI-QTOF): $m / z$ [M + H] $]^{+}$calcd for $\mathrm{C}_{27} \mathrm{H}_{17} \mathrm{O}_{2}$ : 373.1223; found: 373.1220 .

\section{$(10 a S, 14 a R)-10 a, 11,12,13,14,14 a-H e x a h y d r o-10 H-b e n z o[1,2]$ fluo-} reno[3,4-b]benzofuran-10-one (11')

Off-white solid; yield: $22.4 \mathrm{mg}$ (68\%); $\mathrm{mp} 189.3-191.5^{\circ} \mathrm{C}$.

${ }^{1} \mathrm{H}$ NMR $\left(400 \mathrm{MHz}, \mathrm{CDCl}_{3}\right): \delta=9.29(\mathrm{~d}, J=7.7 \mathrm{~Hz}, 1 \mathrm{H}), 8.47(\mathrm{~d}, J=7.7$ $\mathrm{Hz}, 1 \mathrm{H}), 8.04(\mathrm{~d}, J=7.1 \mathrm{~Hz}, 1 \mathrm{H}), 7.67-7.78(\mathrm{~m}, 3 \mathrm{H}), 7.46-7.56(\mathrm{~m}, 2$ H), 3.94-4.02 (m, $1 \mathrm{H}), 3.04$ (br s, $1 \mathrm{H}), 2.52-2.62$ (m, $2 \mathrm{H}), 1.79-1.90$ $(\mathrm{m}, 1 \mathrm{H}), 1.65-1.74(\mathrm{~m}, 2 \mathrm{H}), 1.45-1.54(\mathrm{~m}, 1 \mathrm{H}), 1.28-1.32(\mathrm{~m}, 1 \mathrm{H})$, $1.08-1.17(\mathrm{~m}, 1 \mathrm{H})$.

${ }^{13} \mathrm{C}$ NMR $\left(100 \mathrm{MHz}, \mathrm{CDCl}_{3}\right): \delta=206.7,157.8,156.4,156.3,129.5$, 128.9, 126.9, 126.8, 125.3, 125.1, 124.0, 121.7, 121.4, 120.7, 116.0, $112.4,49.0,38.4,31.4,23.2,22.7,22.4$.

HRMS (ESI-QTOF): $m / z$ [M $+\mathrm{H}]^{+}$calcd for $\mathrm{C}_{23} \mathrm{H}_{19} \mathrm{O}_{2}$ : 327.1380; found: 327.1382 .

\section{Naphtho[1,2-b]benzofuran-5-yl(thiophen-3-yl)methanone (1g)}

Gray solid; yield: $28.6 \mathrm{mg}$ (87\%); $\mathrm{mp} 184.9-186.6^{\circ} \mathrm{C}$.
${ }^{1} \mathrm{H}$ NMR $\left(400 \mathrm{MHz}, \mathrm{CDCl}_{3}\right): \delta=8.54(\mathrm{~d}, J=7.4 \mathrm{~Hz}, 1 \mathrm{H}), 8.36(\mathrm{~d}, J=8.4$ $\mathrm{Hz}, 1 \mathrm{H}), 8.25-8.28(\mathrm{~m}, 1 \mathrm{H}), 7.97(\mathrm{~d}, J=6.1 \mathrm{~Hz}, 1 \mathrm{H}), 7.92(\mathrm{~s}, 1 \mathrm{H})$, 7.68-7.75 (m, $3 \mathrm{H}), 7.48-7.65(\mathrm{~m}, 2 \mathrm{H}), 7.39-7.44(\mathrm{~m}, 2 \mathrm{H})$.

${ }^{13} \mathrm{C}$ NMR $\left(100 \mathrm{MHz}, \mathrm{CDCl}_{3}\right): \delta=191.0,156.4,153.7,143.6,135.4$, $132.8,130.8,128.6,127.5,127.2,126.9,126.7,126.6,124.7,123.6$, $121.8,121.4,121.2,120.5,117.7,112.2$.

HRMS (ESI-QTOF): $\mathrm{m} / z[\mathrm{M}+\mathrm{H}]^{+}$calcd for $\mathrm{C}_{21} \mathrm{H}_{13} \mathrm{O}_{2} \mathrm{~S}: 329.0631$; found: 329.0635 .

\section{2-[(2-Methoxyphenyl)ethynyl]benzaldehyde (12)}

To a solution of 2-iodobenzaldehyde ( $250 \mathrm{mg}, 1.08 \mathrm{mmol}$ ) in $\mathrm{MeCN}$ ( 3 $\mathrm{mL}$ ) were added 1-ethynyl-2-methoxybenzene (157 mg, $1.19 \mathrm{mmol})$, $\left(\mathrm{Ph}_{3} \mathrm{P}\right)_{2} \mathrm{PdCl}_{2}$ (76 mg, $\left.0.108 \mathrm{mmol}\right), \mathrm{CuI}(10 \mathrm{mg}, 0.054 \mathrm{mmol})$, and $\mathrm{Et}_{3} \mathrm{~N}$ $(0.45 \mathrm{~mL}, 3.24 \mathrm{mmol})$. The mixture was stirred at $70{ }^{\circ} \mathrm{C}$ for $2 \mathrm{~h}$, and then it was concentrated in vacuo to yield the crude product. Purification by flash chromatography (silica gel, hexanes/EtOAc, 9:1) afforded 12 (198 mg, 78\%) as an off-white solid; $\mathrm{mp} 80.7-83.2^{\circ} \mathrm{C}$.

${ }^{1} \mathrm{H}$ NMR (400 MHz, CDCl $): \delta=10.74(\mathrm{~s}, 1 \mathrm{H}), 7.95(\mathrm{~d}, J=7.7 \mathrm{~Hz}, 1 \mathrm{H})$, $7.65(\mathrm{~d}, J=7.6 \mathrm{~Hz}, 1 \mathrm{H}), 7.57(\mathrm{t}, J=7.6 \mathrm{~Hz}, 1 \mathrm{H}), 7.52(\mathrm{~d}, J=7.5 \mathrm{~Hz}, 1 \mathrm{H})$, $7.43(\mathrm{t}, J=7.4 \mathrm{~Hz}, 1 \mathrm{H}), 7.35(\mathrm{t}, J=7.8 \mathrm{~Hz}, 1 \mathrm{H}), 6.92-6.98(\mathrm{~m}, 2 \mathrm{H})$, $3.93(\mathrm{~s}, 3 \mathrm{H})$.

${ }^{13} \mathrm{C}$ NMR $\left(100 \mathrm{MHz}, \mathrm{CDCl}_{3}\right): \delta=192.7,160.5,136.0,133.8,133.4$, 133.1, 130.7, 128.5, 127.5, 127.1, 120.7, 111.7, 110.8, 93.2, 89.2, 55.9.

HRMS (ESI-QTOF): $m / z$ [M $+\mathrm{H}]^{+}$calcd for $\mathrm{C}_{16} \mathrm{H}_{13} \mathrm{O}_{2}: 237.0910$; found: 237.0914.

\section{2-[(2-Methoxyphenyl)ethynyl]benzyl Acetate (14)}

To a mixture of 13 (500 mg, $1.81 \mathrm{mmol}$ ) in $\mathrm{Et}_{3} \mathrm{~N}(5 \mathrm{~mL})$ were added 1ethynyl-2-methoxybenzene (251 mg, $1.90 \mathrm{mmol}),\left(\mathrm{Ph}_{3} \mathrm{P}\right)_{2} \mathrm{PdCl}_{2}(127$ $\mathrm{mg}, 0.181 \mathrm{mmol})$, and $\mathrm{CuI}(17 \mathrm{mg}, 0.091 \mathrm{mmol})$. The mixture was stirred at r.t. for $2 \mathrm{~h}$, and then it was concentrated in vacuo to yield the crude product. Purification by flash chromatography (silica gel, hexanes/EtOAc, 9:1) afforded 14 (440 $\mathrm{mg}, 87 \%$ ) as a colorless oil.

${ }^{1} \mathrm{H}$ NMR $\left(400 \mathrm{MHz}, \mathrm{CDCl}_{3}\right): \delta=7.58(\mathrm{~d}, J=6.8 \mathrm{~Hz}, 1 \mathrm{H}), 7.49(\mathrm{~d}, J=7.8$ $\mathrm{Hz}, 1 \mathrm{H}), 7.42-7.43(\mathrm{~m}, 1 \mathrm{H}), 7.29-7.35(\mathrm{~m}, 3 \mathrm{H}), 6.87-6.96(\mathrm{~m}, 2 \mathrm{H})$, $5.41(\mathrm{~s}, 2 \mathrm{H}), 3.93(\mathrm{~s}, 3 \mathrm{H}), 2.13(\mathrm{~s}, 3 \mathrm{H})$.

${ }^{13} \mathrm{C}$ NMR $\left(100 \mathrm{MHz}, \mathrm{CDCl}_{3}\right): \delta=171.1,160.3,137.5,133.3,132.1$, 130.6, 130.1, 128.4, 128.2, 128.1, 123.1, 120.6, 112.4, 110.8, 77.4, 65.2, 55.9, 21.1.

HRMS (ESI-QTOF): $m / z$ [M $+\mathrm{H}]^{+}$calcd for $\mathrm{C}_{18} \mathrm{H}_{17} \mathrm{O}_{3}: 281.1172$; found: 281.1176 .

\section{2-(3-Iodobenzofuran-2-yl)benzyl Acetate (15)}

To a stirred solution of $\mathbf{1 4}(440 \mathrm{mg}, 1.57 \mathrm{mmol})$ in $\mathrm{CH}_{2} \mathrm{Cl}_{2}(10 \mathrm{~mL})$ was added $\mathrm{I}_{2}(1.59 \mathrm{~g}, 6.28 \mathrm{mmol})$ and the resulting mixture was stirred at r.t. for $12 \mathrm{~h}$. Then sat. aq. $\mathrm{Na}_{2} \mathrm{~S}_{2} \mathrm{O}_{3}$ solution was added to the mixture and extracted with $\mathrm{CH}_{2} \mathrm{Cl}_{2}(3 \times 15 \mathrm{~mL})$. The combined organic layers were washed with brine, dried $\left(\mathrm{MgSO}_{4}\right)$, and concentrated in vacuo to yield the crude product. Purification by flash chromatography (silica gel, hexanes/EtOAc, 9:1) afforded $\mathbf{1 5}$ (604 $\mathrm{mg}, \mathbf{9 8 \% )}$ as a colorless gum. ${ }^{1} \mathrm{H} \mathrm{NMR}\left(400 \mathrm{MHz}, \mathrm{CDCl}_{3}\right): \delta=7.68(\mathrm{~d}, J=7.7 \mathrm{~Hz}, 1 \mathrm{H}), 7.45-7.57(\mathrm{~m}, 5$ H), 7.33-7.41 (m, $2 \mathrm{H}), 5.23$ (s, $2 \mathrm{H}), 1.97$ (s, $3 \mathrm{H})$.

${ }^{13} \mathrm{C}$ NMR $\left(100 \mathrm{MHz}, \mathrm{CDCl}_{3}\right): \delta=170.8,154.7,154.6,136.0,131.7$, 131.4, 130.3, 129.3, 129.1, 128.2, 125.9, 123.8, 121.9, 111.5, 65.4, 64.6, 20.9 .

HRMS (ESI-QTOF): $m / z[\mathrm{M}+\mathrm{H}]^{+}$calcd for $\mathrm{C}_{17} \mathrm{H}_{14} \mathrm{IO}_{3}: 392.9982$; found: 392.9981. 


\section{2-(3-Iodobenzofuran-2-yl)benzaldehyde (6)}

To a stirred solution of $\mathbf{1 5}(600 \mathrm{mg}, 1.53 \mathrm{mmol})$ in $\mathrm{MeOH}(50 \mathrm{~mL})$ was added $\mathrm{K}_{2} \mathrm{CO}_{3}(1.06 \mathrm{~g}, 7.65 \mathrm{mmol})$ and the resulting mixture was stirred at r.t. for $30 \mathrm{~min}$. Then the solvent was removed in vacuo. Water was added to the mixture and it was extracted with EtOAc $(3 \times 25$ $\mathrm{mL})$. The combined organic layers were washed with brine, dried $\left(\mathrm{Na}_{2} \mathrm{SO}_{4}\right)$, and concentrated in vacuo to yield the crude product. To the crude product dissolved in $\mathrm{CH}_{2} \mathrm{Cl}_{2}(50 \mathrm{~mL})$ was added PCC $(493 \mathrm{mg}$, $2.29 \mathrm{mmol}$ ) and the mixture was stirred at r.t. for $2 \mathrm{~h}$. Purification by flash chromatography (silica gel, hexanes/EtOAc, 9:1) afforded 6 (439 $\mathrm{mg}, 82 \%)$ as an off-white solid; $\mathrm{mp} 77.4-79.2^{\circ} \mathrm{C}$.

${ }^{1} \mathrm{H}$ NMR (400 MHz, $\left.\mathrm{CDCl}_{3}\right): \delta=10.09(\mathrm{~s}, 1 \mathrm{H}), 8.12(\mathrm{~d}, J=7.5 \mathrm{~Hz}, 1 \mathrm{H})$, $7.82(\mathrm{~d}, J=7.5 \mathrm{~Hz}, 1 \mathrm{H}), 7.74(\mathrm{t}, J=7.5 \mathrm{~Hz}, 1 \mathrm{H}), 7.64(\mathrm{t}, J=7.2 \mathrm{~Hz}, 1 \mathrm{H})$, 7.39-7.51 (m, $4 \mathrm{H})$.

${ }^{13} \mathrm{C}$ NMR $\left(100 \mathrm{MHz}, \mathrm{CDCl}_{3}\right): \delta=191.3,154.96,152.4,134.8,133.6$, 132.6, 131.6, 131.5, 130.3, 128.2, 126.5, 124.1, 122.2, 111.7, 67.9.

HRMS (ESI-QTOF): $m / z$ [M + H] $]^{+}$calcd for $\mathrm{C}_{15} \mathrm{H}_{10} \mathrm{IO}_{2}: 348.9720$; found: 348.9723.

Compound $\mathbf{5}$ was prepared by following the same procedure for the synthesis of $\mathbf{2}$.

\section{2-[3-(Phenylethynyl)benzofuran-2-yl]benzaldehyde (5a)}

Off-white solid; yield: $41.5 \mathrm{mg}$ (92\%); $\mathrm{mp} 102.5-103.6{ }^{\circ} \mathrm{C}$.

${ }^{1} \mathrm{H} \mathrm{NMR}\left(400 \mathrm{MHz}, \mathrm{CDCl}_{3}\right): \delta=10.42(\mathrm{~s}, 1 \mathrm{H}), 8.13(\mathrm{~d}, J=7.8 \mathrm{~Hz}, 1 \mathrm{H})$, $8.06(\mathrm{~d}, J=7.8 \mathrm{~Hz}, 1 \mathrm{H}), 7.82(\mathrm{~d}, J=7.4 \mathrm{~Hz}, 1 \mathrm{H}), 7.74(\mathrm{t}, J=7.6 \mathrm{~Hz}, 1 \mathrm{H})$, 7.52-7.62 (m, $4 \mathrm{H}), 7.36-7.46(\mathrm{~m}, 5 \mathrm{H})$.

${ }^{13} \mathrm{C}$ NMR $\left(100 \mathrm{MHz}, \mathrm{CDCl}_{3}\right): \delta=191.5,154.6,154.4,134.1,133.5$, $132.3,131.7,130.0,129.9,129.1,128.8,128.5,128.2,126.3,123.98$, 122.7, 120.99, 111.7, 104.9, 95.9, 79.7.

HRMS (ESI-QTOF): $m / z$ [M + H] calcd for $\mathrm{C}_{23} \mathrm{H}_{15} \mathrm{O}_{2}$ : 323.1067; found: 323.1065 .

\section{2-[3-( $p$-Tolylethynyl)benzofuran-2-yl]benzaldehyde (5b)}

White solid; yield: $40.0 \mathrm{mg}(85 \%) ; \mathrm{mp} 106.5-108.0^{\circ} \mathrm{C}$.

${ }^{1} \mathrm{H} \mathrm{NMR}\left(400 \mathrm{MHz}, \mathrm{CDCl}_{3}\right): \delta=10.41(\mathrm{~s}, 1 \mathrm{H}), 8.12(\mathrm{~d}, J=7.7 \mathrm{~Hz}, 1 \mathrm{H})$, $8.06(\mathrm{~d}, J=7.8 \mathrm{~Hz}, 1 \mathrm{H}), 7.81(\mathrm{~d}, J=7.3 \mathrm{~Hz}, 1 \mathrm{H}), 7.73(\mathrm{t}, J=7.6 \mathrm{~Hz}, 1 \mathrm{H})$, 7.56-7.61 (m, $2 \mathrm{H}), 7.37-7.45$ (m, $4 \mathrm{H}), 7.16$ (d, $J=7.8 \mathrm{~Hz}, 2 \mathrm{H}), 2.37$ (s, $3 \mathrm{H})$.

${ }^{13} \mathrm{C}$ NMR $\left(100 \mathrm{MHz}, \mathrm{CDCl}_{3}\right): \delta=191.5,154.6,154.2,139.1,134.1$, $133.5,132.3,131.6,130.1,129.8,129.3,129.2,128.2,126.3,123.9$, 121.0, 119.7, 111.7, 105.1, 96.2, 79.0, 21.7.

HRMS (ESI-QTOF): $m / z$ [M + H] $]^{+}$calcd for $\mathrm{C}_{24} \mathrm{H}_{17} \mathrm{O}_{2}$ : 337.1223; found: 337.1226.

\section{2-\{3-[(4-Methoxyphenyl)ethynyl]benzofuran-2-yl\}benzaldehyde (5c)}

Off-white solid; yield: $40.9 \mathrm{mg}$ (83\%); $\mathrm{mp} 140.1-142.2^{\circ} \mathrm{C}$.

${ }^{1} \mathrm{H} \mathrm{NMR}\left(400 \mathrm{MHz}, \mathrm{CDCl}_{3}\right): \delta=10.41(\mathrm{~s}, 1 \mathrm{H}), 8.12(\mathrm{~d}, J=7.6 \mathrm{~Hz}, 1 \mathrm{H})$, $8.04(\mathrm{~d}, J=7.6 \mathrm{~Hz}, 1 \mathrm{H}), 7.81(\mathrm{~d}, J=7.2 \mathrm{~Hz}, 1 \mathrm{H}), 7.73(\mathrm{t}, J=7.2 \mathrm{~Hz}, 1 \mathrm{H})$, 7.56-7.60 (m, $2 \mathrm{H}), 7.38-7.47(\mathrm{~m}, 4 \mathrm{H}), 6.88(\mathrm{~d}, J=8.0 \mathrm{~Hz}, 2 \mathrm{H}), 3.83(\mathrm{~s}$, $3 \mathrm{H})$.

${ }^{13} \mathrm{C}$ NMR $\left(100 \mathrm{MHz}, \mathrm{CDCl}_{3}\right): \delta=191.5,160.1,154.6,153.9,134.1$, 133.5, 133.2, 132.4, 130.0, 129.7, 129.2, 128.2, 126.2, 123.9, 121.0, 114.8, 114.2, 111.7, 105.3, 96.0, 78.3, 55.5.

HRMS (ESI-QTOF): $m / z$ [M + H] calcd for $\mathrm{C}_{24} \mathrm{H}_{17} \mathrm{O}_{3}: 353.1172$; found: 353.1173 .

\section{2-\{3-[(3-Methoxyphenyl)ethynyl]benzofuran-2-yl\}benzaldehyde}

(5d)

Off-white solid; yield: $41.9 \mathrm{mg}$ (85\%); $\mathrm{mp} 121.1-123.0^{\circ} \mathrm{C}$.

${ }^{1} \mathrm{H} \mathrm{NMR}\left(400 \mathrm{MHz}, \mathrm{CDCl}_{3}\right): \delta=10.42(\mathrm{~s}, 1 \mathrm{H}), 8.13(\mathrm{~d}, J=7.8 \mathrm{~Hz}, 1 \mathrm{H})$, $8.02(\mathrm{~d}, J=7.8 \mathrm{~Hz}, 1 \mathrm{H}), 7.82(\mathrm{~d}, J=7.3 \mathrm{~Hz}, 1 \mathrm{H}), 7.74(\mathrm{t}, J=7.5 \mathrm{~Hz}, 1 \mathrm{H})$, 7.57-7.62 (m, 2 H), 7.38-7.46 (m, 2 H), 7.25-7.29 (m, 1 H), 7.12 (d, $J=6.8 \mathrm{~Hz}, 1 \mathrm{H}), 7.05(\mathrm{~s}, 1 \mathrm{H}), 6.91(\mathrm{~d}, J=8.2 \mathrm{~Hz}, 1 \mathrm{H}), 3.85(\mathrm{~s}, 3 \mathrm{H})$.

${ }^{13} \mathrm{C}$ NMR $\left(100 \mathrm{MHz}, \mathrm{CDCl}_{3}\right): \delta=191.5,159.5,154.6,154.5,134.2$, 133.6, 132.3, 130.1, 129.9, 129.6, 129.1, 128.2, 126.3, 124.2, 124.0, 123.7, 121.0, 116.4, 115.5, 111.7, 104.9, 95.8, 79.5, 55.5.

HRMS (ESI-QTOF): $m / z$ [M + H] calcd for $\mathrm{C}_{24} \mathrm{H}_{17} \mathrm{O}_{3}: 353.1172$; found: 353.1171.

\section{2-\{3-[(6-Methoxynaphthalen-2-yl)ethynyl]benzofuran-2-yl\}benz-} aldehyde (5e)

Yellow solid; yield: $42.8 \mathrm{mg}$ (76\%); $\mathrm{mp} 161.1-163.0{ }^{\circ} \mathrm{C}$.

${ }^{1} \mathrm{H} \mathrm{NMR}\left(400 \mathrm{MHz}, \mathrm{CDCl}_{3}\right): \delta=10.46(\mathrm{~s}, 1 \mathrm{H}), 8.15(\mathrm{~d}, J=7.8 \mathrm{~Hz}, 1 \mathrm{H}$ ), $8.09(\mathrm{~d}, J=7.8 \mathrm{~Hz}, 1 \mathrm{H}), 7.97(\mathrm{~s}, 1 \mathrm{H}), 7.86(\mathrm{~d}, J=7.0 \mathrm{~Hz}, 1 \mathrm{H}), 7.69-7.78$ (m, $3 \mathrm{H}), 7.58-7.63(\mathrm{~m}, 2 \mathrm{H}), 7.52(\mathrm{~d}, J=8.4 \mathrm{~Hz}, 1 \mathrm{H}), 7.39-7.47(\mathrm{~m}, 2$ H), $7.17(\mathrm{~d}, J=8.4 \mathrm{~Hz}, 1 \mathrm{H}), 7.12(\mathrm{~s}, 1 \mathrm{H}), 3.94(\mathrm{~s}, 3 \mathrm{H})$.

${ }^{13} \mathrm{C}$ NMR $\left(100 \mathrm{MHz}, \mathrm{CDCl}_{3}\right): \delta=191.6,158.6,154.6,154.2,134.5$, 134.2, 133.6, 132.4, 131.5, 130.1, 129.8, 129.6, 129.2, 128.9, 128.6, 128.3, 127.1, 126.3, 123.98, 121.1, 119.7, 117.6, 111.7, 105.98, 105.1, 96.6, 79.3, 55.5.

HRMS (ESI-QTOF): $m / z$ [M $+\mathrm{H}]^{+}$calcd for $\mathrm{C}_{28} \mathrm{H}_{19} \mathrm{O}_{3}$ : 403.1329; found: 403.1325.

\section{2-\{3-[(3-Fluorophenyl)ethynyl]benzofuran-2-yl\}benzaldehyde (5f)}

Off-white solid; yield: $39.1 \mathrm{mg}$ (82\%); $\mathrm{mp} 129.1-131.2{ }^{\circ} \mathrm{C}$.

${ }^{1} \mathrm{H} \mathrm{NMR}\left(400 \mathrm{MHz}, \mathrm{CDCl}_{3}\right): \delta=10.39(\mathrm{~s}, 1 \mathrm{H}), 8.13(\mathrm{~d}, J=7.7 \mathrm{~Hz}, 1 \mathrm{H})$, $8.03(\mathrm{~d}, J=7.8 \mathrm{~Hz}, 1 \mathrm{H}), 7.80(\mathrm{~d}, J=7.3 \mathrm{~Hz}, 1 \mathrm{H}), 7.75(\mathrm{t}, J=7.4 \mathrm{~Hz}, 1 \mathrm{H})$, 7.58-7.64 (m, 2 H), 7.38-7.47 (m, 2 H), 7.30-7.35 (m, 2 H), 7.21 (d, $J=9.2 \mathrm{~Hz}, 1 \mathrm{H}), 7.06(\mathrm{t}, J=7.8 \mathrm{~Hz}, 1 \mathrm{H})$.

${ }^{13} \mathrm{C}$ NMR $\left(100 \mathrm{MHz}, \mathrm{CDCl}_{3}\right): \delta=191.4,163.7,161.3,154.8,154.6$, 134.2, 133.6, 132.1, 130.2, 130.14, 130.08, 130.0, 128.9, 128.3, 127.60, 127.57, 126.4, 124.1, 120.9, 118.5, 118.3, 116.3, 116.1, 111.8, 104.6, 94.59, 94.56, 80.7.

HRMS (ESI-QTOF): $m / z[\mathrm{M}+\mathrm{H}]^{+}$calcd for $\mathrm{C}_{23} \mathrm{H}_{14} \mathrm{FO}_{2}$ : 341.0972; found: 341.0977.

\section{2-[3-(Cyclohex-1-en-1-ylethynyl)benzofuran-2-yl]benzaldehyde} (5g)

Colorless oil; yield: $40.2 \mathrm{mg}$ (88\%).

${ }^{1} \mathrm{H}$ NMR (400 MHz, $\mathrm{CDCl}_{3}$ ): $\delta=10.34(\mathrm{~s}, 1 \mathrm{H}), 8.09$ (d, $J=7.8 \mathrm{~Hz}, 1 \mathrm{H}$ ), 8.00 (d, J = 7.8 Hz, $1 \mathrm{H}), 7.69-7.74(\mathrm{~m}, 2 \mathrm{H}), 7.53-7.59$ (m, $2 \mathrm{H}), 7.35-$ 7.42 (m, 2 H), 6.23-6.25 (m, 1 H), 2.12-2.21 (m, 4 H), 1.61-1.71 (m, 4 $\mathrm{H})$.

${ }^{13} \mathrm{C}$ NMR $\left(100 \mathrm{MHz}, \mathrm{CDCl}_{3}\right): \delta=191.5,154.5,153.6,136.3,133.97$, $133.5,132.4,129.9,129.6,129.2,128.1,126.1,123.8,120.98,120.5$, 111.6, 105.4, 97.97, 28.9, 25.9, 22.3, 21.6.

HRMS (ESI-QTOF): $m / z$ [M $+\mathrm{H}]^{+}$calcd for $\mathrm{C}_{23} \mathrm{H}_{19} \mathrm{O}_{2}: 327.1380$; found: 327.1383.

\section{2-[3-(Hex-1-yn-1-yl)benzofuran-2-yl]benzaldehyde (5h)}

Colorless oil; yield: $40.6 \mathrm{mg}$ (96\%). 
${ }^{1} \mathrm{H} \mathrm{NMR}\left(400 \mathrm{MHz}, \mathrm{CDCl}_{3}\right): \delta=10.32(\mathrm{~s}, 1 \mathrm{H}), 8.09(\mathrm{dd}, J=0.8,7.8 \mathrm{~Hz}, 1$ H), 7.99 (d, $J=7.8 \mathrm{~Hz}, 1 \mathrm{H}), 7.68-7.72(\mathrm{~m}, 2 \mathrm{H}), 7.52-7.59(\mathrm{~m}, 2 \mathrm{H})$, 7.33-7.41 (m, 2 H), $2.45(\mathrm{t}, J=7.0 \mathrm{~Hz}, 2 \mathrm{H}), 1.57-1.61(\mathrm{~m}, 2 \mathrm{H}), 1.43-$ $1.48(\mathrm{~m}, 2 \mathrm{H}), 0.94(\mathrm{t}, J=7.3 \mathrm{~Hz}, 2 \mathrm{H})$.

${ }^{13} \mathrm{C}$ NMR $\left(100 \mathrm{MHz}, \mathrm{CDCl}_{3}\right): \delta=191.5,154.5,153.7,133.97,133.4$, 132.5, 129.9, 129.6, 129.5, 128.0, 126.0, 123.7, 120.9, 111.6, 105.5, 97.7, 70.7, 30.6, 22.1, 19.5, 13.7.

HRMS (ESI-QTOF): $m / z[\mathrm{M}+\mathrm{Na}]^{+}$calcd for $\mathrm{C}_{21} \mathrm{H}_{18} \mathrm{NaO}_{2}: 325.1199$; found: 325.1194 .

\section{2-[3-(Thiophen-3-ylethynyl)benzofuran-2-yl]benzaldehyde (5i)}

Yellow solid; yield: $44.1 \mathrm{mg}$ (96\%); mp $120.6-121.9{ }^{\circ} \mathrm{C}$.

${ }^{1} \mathrm{H}$ NMR $\left(400 \mathrm{MHz}, \mathrm{CDCl}_{3}\right): \delta=10.40(\mathrm{~s}, 1 \mathrm{H}), 8.11(\mathrm{~d}, J=7.7 \mathrm{~Hz}, 1 \mathrm{H})$, $8.03(\mathrm{~d}, J=7.8 \mathrm{~Hz}, 1 \mathrm{H}), 7.80(\mathrm{~d}, J=7.5 \mathrm{~Hz}, 1 \mathrm{H}), 7.73(\mathrm{t}, J=7.5 \mathrm{~Hz}, 1 \mathrm{H})$, 7.57-7.61 (m, 3 H), 7.37-7.45 (m, 2 H), 7.30-7.32 (m, 1 H), 7.19 (d, $J=4.8 \mathrm{~Hz}, 1 \mathrm{H})$.

${ }^{13} \mathrm{C}$ NMR $\left(100 \mathrm{MHz}, \mathrm{CDCl}_{3}\right): \delta=191.4,154.6,154.3,134.1,133.6$, $132.3,130.0,129.9,129.8,129.5,129.0,128.2,126.3,125.7,123.98$, 121.7, 121.0, 111.7, 104.99, 91.1, 79.2.

HRMS (ESI-QTOF): $m / z[\mathrm{M}+\mathrm{H}]^{+}$calcd for $\mathrm{C}_{21} \mathrm{H}_{13} \mathrm{O}_{2} \mathrm{~S}$ : 329.0631 ; found: 329.0651

Compound $\mathbf{4}$ was prepared by following the same procedure for the synthesis of $\mathbf{1}$.

\section{Naphtho[1,2-b]benzofuran-6-yl(phenyl)methanone (4a)}

Yellow solid; yield: $29.0 \mathrm{mg}(90 \%) ; \mathrm{mp} 152.9-154.6{ }^{\circ} \mathrm{C}$.

${ }^{1} \mathrm{H}$ NMR $\left(400 \mathrm{MHz}, \mathrm{CDCl}_{3}\right): \delta=8.53(\mathrm{~d}, J=8.3 \mathrm{~Hz}, 1 \mathrm{H}), 7.97-8.05(\mathrm{~m}, 5$ H), 7.73-7.78 (m, $2 \mathrm{H}), 7.61-7.68(\mathrm{~m}, 2 \mathrm{H}), 7.47-7.56(\mathrm{~m}, 3 \mathrm{H}), 7.31(\mathrm{t}$, $J=7.4 \mathrm{~Hz}, 1 \mathrm{H})$.

${ }^{13} \mathrm{C}$ NMR $\left(100 \mathrm{MHz}, \mathrm{CDCl}_{3}\right): \delta=196.4,156.5,152.8,137.99,133.3$, 131.5, 131.2, 130.7, 129.5, 128.7, 128.6, 127.2, 127.1, 126.9, 124.0, 123.2, 122.8, 121.3, 117.6, 111.7 .

HRMS (ESI-QTOF): $m / z[\mathrm{M}+\mathrm{H}]^{+}$calcd for $\mathrm{C}_{23} \mathrm{H}_{15} \mathrm{O}_{2}$ : 323.1067; found: 323.1065 .

\section{Naphtho[1,2-b]benzofuran-6-yl (p-tolyl)methanone (4b)}

White solid; yield: $32.0 \mathrm{mg}$ (95\%); $\mathrm{mp} 146.8-148.5{ }^{\circ} \mathrm{C}$.

${ }^{1} \mathrm{H} \mathrm{NMR}\left(400 \mathrm{MHz}, \mathrm{CDCl}_{3}\right): \delta=8.53(\mathrm{~d}, J=8.3 \mathrm{~Hz}, 1 \mathrm{H}), 7.98-8.01(\mathrm{~m}, 2$ H), 7.91-7.95 (m, $3 \mathrm{H}), 7.72-7.77(\mathrm{~m}, 2 \mathrm{H}), 7.62(\mathrm{t}, J=7.2 \mathrm{~Hz}, 1 \mathrm{H}), 7.48$ $(\mathrm{t}, J=7.8 \mathrm{~Hz}, 1 \mathrm{H}), 7.28-7.34(\mathrm{~m}, 3 \mathrm{H}), 2.48(\mathrm{~s}, 3 \mathrm{H})$.

${ }^{13} \mathrm{C}$ NMR $\left(100 \mathrm{MHz}, \mathrm{CDCl}_{3}\right): \delta=196.1,156.4,152.8,144.3,135.3$, $130.9,129.44,129.39,128.5,127.1,126.8,126.7,124.0,123.9,123.2$, 121.3, 111.7, 21.9.

HRMS (ESI-QTOF): $m / z[\mathrm{M}+\mathrm{H}]^{+}$calcd for $\mathrm{C}_{24} \mathrm{H}_{17} \mathrm{O}_{2}$ : 337.1223; found: 337.1228 .

\section{(4-Methoxyphenyl)(naphtho[1,2-b]benzofuran-6-yl)methanone \\ (4c)}

Off-white solid; yield: $31.7 \mathrm{mg}$ (90\%); $\mathrm{mp} 154.5-156.2^{\circ} \mathrm{C}$.

${ }^{1} \mathrm{H} \mathrm{NMR}\left(400 \mathrm{MHz}, \mathrm{CDCl}_{3}\right): \delta=8.52(\mathrm{~d}, J=8.2 \mathrm{~Hz}, 1 \mathrm{H}), 7.99-8.02(\mathrm{~m}, 3$ H), 7.91-7.93 (m, $2 \mathrm{H}), 7.71-7.77(\mathrm{~m}, 2 \mathrm{H}), 7.63(\mathrm{t}, J=7.6 \mathrm{~Hz}, 1 \mathrm{H}), 7.47$ $(\mathrm{t}, J=7.3 \mathrm{~Hz}, 1 \mathrm{H}), 7.29(\mathrm{t}, J=7.8 \mathrm{~Hz}, 1 \mathrm{H}), 7.00(\mathrm{~d}, J=8.8 \mathrm{~Hz}, 2 \mathrm{H}), 3.91$ (s, $3 \mathrm{H})$.

${ }^{13} \mathrm{C}$ NMR $\left(100 \mathrm{MHz}, \mathrm{CDCl}_{3}\right): \delta=195.1,163.98,156.4,152.7,133.1$, 131.64, 131.63, 130.6, 129.3, 128.3, 127.1, 126.8, 126.0, 124.0, 123.7, $123.2,122.6,121.3,117.5,114.0,111.7,55.7$.
HRMS (ESI-QTOF): $m / z$ [M $+\mathrm{H}]^{+}$calcd for $\mathrm{C}_{24} \mathrm{H}_{17} \mathrm{O}_{3}$ : 353.1172; found: 353.1175 .

\section{(3-Methoxyphenyl)(naphtho[1,2-b]benzofuran-6-yl)methanone} (4d)

Brown solid, yield: $31.0 \mathrm{mg}$ (88\%); $\mathrm{mp} 137.9-139.5^{\circ} \mathrm{C}$.

${ }^{1} \mathrm{H}$ NMR $\left(400 \mathrm{MHz}, \mathrm{CDCl}_{3}\right): \delta=8.53(\mathrm{~d}, J=8.2 \mathrm{~Hz}, 1 \mathrm{H}), 8.06$ (d, $J=7.8$ $\mathrm{Hz}, 1 \mathrm{H}), 7.98-8.00$ (m, $2 \mathrm{H}), 7.73-7.77(\mathrm{~m}, 2 \mathrm{H}), 7.61-7.64(\mathrm{~m}, 2 \mathrm{H})$, 7.47-7.53 (m, $2 \mathrm{H}), 7.41(\mathrm{t}, J=7.9 \mathrm{~Hz}, 1 \mathrm{H}), 7.32(\mathrm{t}, J=7.5 \mathrm{~Hz}, 1 \mathrm{H})$, $7.21(\mathrm{~d}, J=8.1 \mathrm{~Hz}, 1 \mathrm{H}), 3.89$ (s, $3 \mathrm{H})$.

${ }^{13} \mathrm{C}$ NMR $\left(100 \mathrm{MHz}, \mathrm{CDCl}_{3}\right): \delta=196.2,159.9,156.5,152.8,139.3$, $131.4,131.1,129.6,129.5,128.6,127.2,127.1,126.9,123.97,123.8$, 123.2, 122.8, 121.3, 120.0, 117.5, 114.4, 111.7, 55.7 .

HRMS (ESI-QTOF): $m / z[\mathrm{M}+\mathrm{Na}]^{+}$calcd for $\mathrm{C}_{24} \mathrm{H}_{16} \mathrm{NaO}_{3}: 375.0992$; found: 375.0993 .

\section{6-Methoxynaphthalen-1-yl(naphtho[1,2-b]benzofuran-6- yl)methanone (4e)}

Off-white solid; yield: $35.8 \mathrm{mg}$ (89\%); $\mathrm{mp} 161.3-162.6^{\circ} \mathrm{C}$.

${ }^{1} \mathrm{H} \mathrm{NMR}\left(400 \mathrm{MHz}, \mathrm{CDCl}_{3}\right): \delta=8.56(\mathrm{~d}, J=7.9 \mathrm{~Hz}, 1 \mathrm{H}), 8.36(\mathrm{~s}, 1 \mathrm{H})$, $8.16(\mathrm{~d}, J=8.4 \mathrm{~Hz}, 1 \mathrm{H}), 7.99-8.01(\mathrm{~m}, 3 \mathrm{H}), 7.88(\mathrm{~d}, J=8.5 \mathrm{~Hz}, 1 \mathrm{H})$, 7.73-7.77 (m, $3 \mathrm{H}), 7.62-7.66(\mathrm{~m}, 1 \mathrm{H}), 7.47(\mathrm{t}, J=7.8 \mathrm{~Hz}, 1 \mathrm{H}), 7.29$ (d, $J=7.8 \mathrm{~Hz}, 1 \mathrm{H}), 7.18-7.22(\mathrm{~m}, 2 \mathrm{H}), 3.97(\mathrm{~s}, 3 \mathrm{H})$.

${ }^{13} \mathrm{C}$ NMR $\left(100 \mathrm{MHz}, \mathrm{CDCl}_{3}\right): \delta=196.2,160.1,156.5,152.8,137.6$, 133.1, 133.0, 131.6, 131.4, 129.4, 128.4, 127.8, 127.5, 127.1, 126.8, 126.6, 124.0, 123.8, 123.3, 122.7, 121.3, 119.98, 117.7, 111.7, 105.98, 55.6.

HRMS (ESI-QTOF): $m / z$ [M $+\mathrm{H}]^{+}$calcd for $\mathrm{C}_{28} \mathrm{H}_{19} \mathrm{O}_{3}$ : 403.1329; found: 403.1327.

(3-Fluorophenyl)(naphtho[1,2-b]benzofuran-6-yl)methanone (4f) Off-white solid; yield: $30.6 \mathrm{mg}$ (90\%); mp 130.7-132.6 ${ }^{\circ} \mathrm{C}$.

${ }^{1} \mathrm{H} \mathrm{NMR}\left(400 \mathrm{MHz}, \mathrm{CDCl}_{3}\right): \delta=8.54(\mathrm{~d}, J=8.2 \mathrm{~Hz}, 1 \mathrm{H}), 8.06(\mathrm{~d}, J=7.9$ $\mathrm{Hz}, 1 \mathrm{H}), 8.00$ (d, J = 8.2 Hz, $1 \mathrm{H}), 7.97(\mathrm{~s}, 1 \mathrm{H}), 7.72-7.80(\mathrm{~m}, 4 \mathrm{H}), 7.64$ $(\mathrm{t}, J=7.5 \mathrm{~Hz}, 1 \mathrm{H}), 7.48-7.52(\mathrm{~m}, 2 \mathrm{H}), 7.31-7.39(\mathrm{~m}, 2 \mathrm{H})$.

${ }^{13} \mathrm{C}$ NMR $\left(100 \mathrm{MHz}, \mathrm{CDCl}_{3}\right): \delta=195.0,164.1,161.6,156.5,152.9$, $140.2,140.1,131.4,130.6,130.3,129.5,128.9,127.5,127.3,127.0$, $126.6,123.95,123.89,123.3,123.0,121.4,120.4,120.2,117.5,117.3$, $117.1,111.8,55.6$.

HRMS (ESI-QTOF): $m / z[\mathrm{M}+\mathrm{H}]^{+}$calcd for $\mathrm{C}_{23} \mathrm{H}_{14} \mathrm{FO}_{2}: 341.0972$; found: 341.0977 .

(9cS,13aR)-9c,10,11,12,13,13a-Hexahydro-14H-benzo[3,4]fluoreno[2,1-b]benzofuran-14-one $\left(\mathbf{4 g}^{\prime}\right)$

Colorless oil; yield: $26.8 \mathrm{mg}(82 \%)$.

${ }^{1} \mathrm{H} \mathrm{NMR}\left(400 \mathrm{MHz}, \mathrm{CDCl}_{3}\right): \delta=8.91(\mathrm{~d}, J=7.4 \mathrm{~Hz}, 1 \mathrm{H}), 8.55(\mathrm{~d}, J=8.2$ $\mathrm{Hz}, 1 \mathrm{H}), 8.24(\mathrm{~d}, J=8.1 \mathrm{~Hz}, 1 \mathrm{H}), 7.81(\mathrm{t}, J=7.3 \mathrm{~Hz}, 1 \mathrm{H}), 7.67-7.72(\mathrm{~m}$, $2 \mathrm{H}), 7.52(\mathrm{t}, J=7.5 \mathrm{~Hz}, 1 \mathrm{H}), 7.45(\mathrm{t}, J=7.2 \mathrm{~Hz}, 1 \mathrm{H}), 4.02-4.08(\mathrm{~m}, 1$ H), 3.05 (br s, $1 \mathrm{H}), 2.53-2.61(\mathrm{~m}, 2 \mathrm{H}), 1.82-1.88(\mathrm{~m}, 1 \mathrm{H}), 1.65-1.76$ $(\mathrm{m}, 2 \mathrm{H}), 1.42-1.51(\mathrm{~m}, 1 \mathrm{H}), 1.22-1.31(\mathrm{~m}, 1 \mathrm{H}), 1.01-1.11(\mathrm{~m}, 1 \mathrm{H})$.

${ }^{13} \mathrm{C}$ NMR $\left(100 \mathrm{MHz}, \mathrm{CDCl}_{3}\right): \delta=207.1,156.51,156.49,152.1,129.3$, $128.6,128.4,127.1,126.9,125.41,125.39,124.5,124.3,123.5,122.3$, $115.1,111.4,49.4,38.6,33.8,23.8,23.0,22.9$.

HRMS (ESI-QTOF): $m / z$ [M $+\mathrm{H}]^{+}$calcd for $\mathrm{C}_{23} \mathrm{H}_{19} \mathrm{O}_{2}: 327.1380$; found: 327.1384 . 


\section{1-Naphtho[1,2-b]benzofuran-6-yl)pentan-1-one (4h)}

Off-white solid; yield: $29.3 \mathrm{mg}(97 \%) ; \mathrm{mp} 134.6-136.2^{\circ} \mathrm{C}$.

${ }^{1} \mathrm{H} \mathrm{NMR}\left(400 \mathrm{MHz}, \mathrm{CDCl}_{3}\right): \delta=8.76(\mathrm{~d}, J=7.9 \mathrm{~Hz}, 1 \mathrm{H}), 8.65(\mathrm{~d}, J=7.8$ $\mathrm{Hz}, 1 \mathrm{H}), 7.76(\mathrm{~d}, J=7.8 \mathrm{~Hz}, 1 \mathrm{H}), 7.63-7.72(\mathrm{~m}, 4 \mathrm{H}), 7.49-7.53(\mathrm{~m}, 1$ H), 7.43-7.46 (m, $1 \mathrm{H}), 2.85(\mathrm{t}, J=7.7 \mathrm{~Hz}, 2 \mathrm{H}), 1.66-1.70(\mathrm{~m}, 2 \mathrm{H})$, $1.45-1.51(\mathrm{~m}, 2 \mathrm{H}), 0.98(\mathrm{t}, J=7.3 \mathrm{~Hz}, 3 \mathrm{H})$.

${ }^{13} \mathrm{C}$ NMR $\left(100 \mathrm{MHz}, \mathrm{CDCl}_{3}\right): \delta=182.98,156.7,153.9,146.1,137.9$, 134.6, 132.9, 130.2, 129.7, 127.6, 127.4, 127.2, 126.6, 125.0, 124.6, $122.2,110.7,35.8,32.1,23.0,14.2$.

HRMS (ESI-QTOF): $m / z$ [M $+\mathrm{H}]^{+}$calcd for $\mathrm{C}_{21} \mathrm{H}_{19} \mathrm{O}_{2}$ : 303.1380; found: 303.1378.

\section{Naphtho[1,2-b]benzofuran-6-yl(thiophen-3-yl)methanone (4i)}

Gray solid; yield: $28.6 \mathrm{mg}$ (87\%); $\mathrm{mp} 137.8-139.6^{\circ} \mathrm{C}$

${ }^{1} \mathrm{H} \mathrm{NMR}\left(400 \mathrm{MHz}, \mathrm{CDCl}_{3}\right): \delta=8.52(\mathrm{~d}, J=7.6 \mathrm{~Hz}, 1 \mathrm{H}), 8.02-8.09(\mathrm{~m}, 4$ $\mathrm{H}), 7.72-7.78(\mathrm{~m}, 3 \mathrm{H}), 7.64(\mathrm{t}, J=6.8 \mathrm{~Hz}, 1 \mathrm{H}), 7.47-7.50(\mathrm{~m}, 2 \mathrm{H}), 7.33$ $(\mathrm{t}, J=6.8 \mathrm{~Hz}, 1 \mathrm{H})$.

${ }^{13} \mathrm{C}$ NMR $\left(100 \mathrm{MHz}, \mathrm{CDCl}_{3}\right): \delta=189.7,156.4,152.8,142.3,135.5$, 131.96, 131.5, 129.4, 128.63, 128.56, 127.1, 126.9, 126.8, 126.2, 123.9, $123.3,122.8,121.3,117.2,111.7$.

HRMS (ESI-QTOF): $m / z[\mathrm{M}+\mathrm{H}]^{+}$calcd for $\mathrm{C}_{21} \mathrm{H}_{13} \mathrm{O}_{2} \mathrm{~S}: 329.0631$; found: 329.0635 .

\section{Acknowledgement}

This work was supported by the National Research Foundation of Korea (NRF) grant funded by the Korea government (MSIP) (2014R1A2A1A11050491).

\section{Supporting Information}

Supporting Information for this article is available online at http://dx.doi.org/10.1055/s-0036-1588120. ${ }^{1} \mathrm{H}$ and ${ }^{13} \mathrm{C}$ NMR spectra of synthesized compounds are included.

\section{References}

(1) For reviews, see: (a) Liu, L.; Xu, B.; Hammond, G. B. Beilstein J. Org. Chem. 2011, 7, 606. (b) Saito, A.; Tateishi, K. Heterocycles 2016, 92, 607.

(2) Nayak, M.; Kim, I. Org. Biomol. Chem. 2015, 13, 9697.

(3) (a) Jung, Y.; Kim, I. J. Org. Chem. 2015, 80, 2001. (b) Nayak, M.; Kim, I. J. Org. Chem. 2015, 80, 11460.

(4) (a) Hamasaki, A.; Zimpleman, J. M.; Hwang, I.; Boger, D. L. J. Am. Chem. Soc. 2005, 127, 10767. (b) Liu, Z.; Larock, R. C. J. Org. Chem. 2007, 72, 223. (c) Shi, Z.; Ding, S.; Cui, Y.; Jiao, N. Angew. Chem. Int. Ed. 2009, 48, 7895. (d) Morin, M.; Levesque, P.; Barriault, L. Beilstein J. Org. Chem. 2013, 9, 2625. (e) Rao, H. S. P.; Vijjapu, S. RSC Adv. 2014, 4, 25747. (f) Suh, S.-E.; Barros, S. A.; Chenoweth, D. M. Chem. Sci. 2015, 6, 5128. (g) Saito, K.; Chikkade, P. K.; Kanai, M.; Kuninobu, Y. Chem. Eur. J. 2015, 21, 8365. (h) Wang, S.; Lv, B.; Cui, Q.; Ma, X.; Ba, X.; Xiao, J. Chem. Eur. J. 2015, 21, 14791.

(5) (a) Kim, I.; Lee, S.-H.; Lee, S. Tetrahedron Lett. 2008, 49, 6579. (b) Kim, I.; Choi, J. Org. Biomol. Chem. 2009, 7, 2788. (c) Kim, I.; Kim, K.; Choi, J. J. Org. Chem. 2009, 74, 8492. (d) Kim, K.; Kim, I. Org. Lett. 2010, 12, 5314. (e) Lee, S.-H.; Kim, I.; Kim, S. H. Bull. Korean Chem. Soc. 2011, 32, 4137. (f) Lee, J. H.; Kim, M.; Kim, I. J. Org. Chem. 2014, 79, 6153. (g) Nayak, M.; Jung, Y.; Kim, I. Org. Biomol. Chem. 2016, 14, 8074. (h) Jung, Y.; Kim, I. Org. Biomol. Chem. 2016, 14, 10454.

(6) (a) Huang, X.-C.; Wang, F.; Liang, Y.; Li, J.-H. Org. Lett. 2009, 11, 1139. (b) Byers, P. M.; Rashid, J. I.; Mohamed, R. K.; Alabugin, I. V. Org. Lett. 2012, 14, 6032. (c) Yamamoto, Y.; Matsui, K.; Shibuya, M. Chem. Eur. J. 2015, 21, 7245. (d) Ngo, T. N.; Dang, T. T.; Villinger, A.; Langer, P. Adv. Synth. Catal. 2016, 358, 1328.

(7) 5- or 6-Acylnaphtho[1,2-b]benzofuran can be viewed as a hybrid molecule consisting of 2-arylbenzofuran and 1- or 2acylnaphthalene.

(8) (a) Tietze, L. F.; Bell, H. P.; Chandrasekhar, S. Angew. Chem. Int. Ed. 2003, 42, 3996. (b) Kouznetsov, V. V.; Gomez-Barrio, A. Eur. J. Med. Chem. 2009, 44, 3091. (c) Shaveta, S. M.; Palwinder, S. Eur. J. Med. Chem. 2016, 124, 500.

(9) Kang, B.; Lee, M. H.; Kim, M.; Hwang, J.; Kim, H. B.; Chi, D. Y. J. Org. Chem. 2015, 80, 8254.

(10) Neises, B.; Steglich, W. Angew. Chem., Int. Ed. Engl. 1978, 17, 522.

(11) (a) Jin, T.; Yamamoto, Y. Org. Lett. 2008, 10, 3137. (b) Jin, T.; Yang, F.; Liu, C.; Yamamoto, Y. Chem. Commun. 2009, 3533.

(12) Mehta, S.; Waldo, J. P.; Larock, R. C. J. Org. Chem. 2009, 74, 1141. 